\title{
The First Half-Century of Decision Modelling for Substitutable Products: A Literature Review and Bibliographic Analysis
}

\author{
Gaurav Nagpal \\ Birla Institute of Science and Technology, Pilani, India \\ Email: gaurav19821@gmail.com (Corresponding Author) \\ Udayan Chanda \\ Birla Institute of Science and Technology, Pilani, India \\ Email: udayanchanda@pilani.bits-pilani.ac.in \\ Himanshu Seth \\ Birla Institute of Science and Technology, Pilani, India \\ Email: hseth91@gmail.com
}

\begin{abstract}
Due to the resource constraints of the factors of production (land, capital, capacity, etc.), the firms have to rationalize the assortment of products that they deal in. In such a situation, a consumer whose requirement for a preferred product may not get served from the existing inventories with the seller, may go for some other alternative product. A lot of research work in the past has been done on this demand substitution and its implications upon the assortment optimization, inventory optimization and price optimization. The first work on modelling for substitutable demand is attributed to 1970. Considering the same, the authors decided to do a review of the half-century of work on the modelling for substitutable products. The paper gives a detailed analysis of the published research articles around demand substitution and also discusses the achievements of the existing research. The article also gives a few possibilities of the future extensions to this body of knowledge on the management of substitutable products.
\end{abstract}

Keywords: demand substitution, substitutable products, inventory optimization, pricing optimization, assortment optimization, demand estimation, technological substitution

\section{INTRODUCTION}

In the retail environment, the needs of a customer can be served by any of the multiple products (Nagasawa et al. 2014). Considering this demand substitution in serving the customer influences the stocking decisions. If a few of the items are understocked, the resulting demand substitution leads to an increase in the demand for other items, and thus, changing the optimal inventory decisions. The product assortment decisions are particularly very important in a retail scenario with limited shelf space. Ignoring the substitution of consumer demand in these situations can lead to inefficient or sub-optimal assortments. Optimal assortment planning helps a retailer in deciding which items to carry and in what quantity. Demand substitution is the use of one product to serve the demand for some other product belonging to the same product category (Chopra and Meindl,
2010). The product category is defined as a set of similar products having alternative attributes like features, form, shape, colour, size, specifications, etc. The domain of product substitution has received good focus in Operations Management because of the influence it carries upon the effectiveness of the decisions made by the firms on the inventory planning, production planning and control, product basket rationalization, pricing, etc (Rajaram and Tang 2001, Ganesh et al. 2008, Goyal and Netessine 2011).

Many of the industries have been successfully practising something that is called 'demand conditioning' by constantly changing the product value proposition (in terms of pricing and configuration) to influence customer demand (An and Ramachandran 2005, Song and Xue 2007). Song and Xue (2007) pointed out that companies like 'Dell Computers' are also practising 'demand conditioning' based on present inventory levels to influence the potential customer. They consider the current inventory levels while practising this concept and thereby, aim to ensure high inventory velocity. Smith and Agrawal (2000) suggested that while offering a variety of products has its advantages however it will also increase the inventory-related cost. Thus firms' should optimize their assortments of product and accordingly plan the inventory levels of each item.

The emergence of big data revolution has also enabled the availability and processing of huge demand data of substitutable products (Yudhisytra et al. 2020) so that the managers can make sound business decisions. The number of variants of any product has increased immensely over some time in the pursuit of mass customization. This has led to tremendous scope for optimization of business decisions using data analytics and quantitative modelling. Some of the common examples of quantitative methods that can be seen in the daily life of a business are markdown optimization by the retailers, shelf space planning in the retail industry, capacity planning and inventory planning.

With the importance that the topic of demand substitution modelling has gained, It is very important to collate the existing literature under these domains for the substitutable products. Although new knowledge is getting 
generated in this domain at a fast pace, it is fragmented and multi-disciplinary. This makes it challenging for the researchers to keep themselves up to date with the latest research. That is the reason this review has been done to enable the research scholars to do a fair assessment of the collective work.

Demand substitution of products is a double-edged sword because it provides the opportunities of inventory pooling while increasing the uncertainty around the expected demand of each variant. Demand substitution not only influences the supply chain relationships downstream of the manufacturer but also influences the decisions upstream of the manufacturer. The substitution of a product by another can be because of multiple reasons such as the launch of a new product in the assortment, or stocking out of the customer's first choice, or the sudden jump in the price of the first choice product, or increase in the technology acceptance or technology needs of the customer leading him to switch to a more advanced product within the same category. Netessine and Rudi (2003) discussed an optimal stocking of substitutable products using a consumer-driven substitution model under competition for a random number of products. Chen and Plambeck (2008) suggested that the inventory level of substitutable products also affects the demand and buying behaviour and hence it is important to know the demand distribution and substitution pattern. Thus store footprint can be reduced and assortment can be downsized by estimating the substitution effects in a retail basket.

Also, the substitution is sometimes propelled by the vendors, and many a time by the customers. While some situations are characterised by uni-directional substitution, other situations may be a bi-directional substitution. The nature of choice decisions is also different in the literature with three popular choice phenomenon being multinomial logit, bayesian logit, markovian and few other location choice models (Timmermans and Golledge 1990). The objective of this review paper is to classify the research papers based on the nature of demand substitution, the propelling agent behind the substitution and the direction of substitution. This paper presents a comprehensive review and discussion on inventory models based on varied type of substitution models that are available in the literature. Also, solution approaches used to solve these substitutions-based model will be discussed in detail.

This literature review intends to document and analyse literature relating to the integration and implementation of supply chain management practices. As such, it is organized into the following sub-sections. In Section 2, the methodology considered for selection of papers has been discussed, and the papers written in the last five decades have been classified into broad categories as per the objective of the study considered. In Section 3, a few important papers related to substitution demand pattern published in the latest two decades have been discussed in greater detail. In Section 4 , a detailed classification of the existing work has been done not only in terms of the objective of the study but also by the nature of substitution, the direction of substitution and the propelling force behind the substitution. In Section 5, the existing literature has been classified based on optimization methods used. Section 6 illustrates the statistics of the existing research done on modelling for substitutable products. Section 7 serves to summarize the accomplishments of the work done in the five decades. Section 8 outlines the limitations of the existing research, introduces the directions for further research in modelling for substitutable demand, and captures the conclusions of the paper.

\section{METHODOLOGY AND ANALYSIS OF INVENTORY PAPERS RELATED TO SUBSTITUTION DEMAND PATTERN}

This section discusses the methodology adopted for performing the review and the outcome of the review in terms of analysis of the comprehensive research papers on the theme.

\subsection{Research Methodology}

This is a semi-centennial review on the models developed for the products with substitutable demand. Since Scopus is generally considered to be the most popular and comprehensive database of research articles, the review began with the keyword search of "demand substitution" in the Scopus database, and 7,505 document results were obtained. Then, the authors limited themselves to the area of "Business, Management and Accounting" and the list was filtered down to 760 documents. Now, these papers had multiple themes such as business implications (pricing, inventory, assortment decisions, etc.), environmental implications, trade implications, sustainability implications, etc.) Also, while some dealt in demand substitution of physical goods, others dealt in the demand substitution of currency, services, energy, fuels, etc. Only those research papers were considered that deal in the business and management implications for demand substitution of the physical products and selected 156 papers. Also, the studies that were pure empirical studies were excluded since our emphasis was on the modelling for substitutable items. Citation chaining was also done and the references of the articles were mined to search for all the useful references. Preference was given to the articles published in the journals that are rated by ABDC (Australian Business Dean's Council) to ensure the quality of the review. It was the endeavour that the review is very trustworthy, rigorous and thorough. The selected papers were given a thorough reading to come up with the insights on the key themes, research objectives, nature of substitution, model types and the solution approaches proposed by these articles. The guidelines by Torraco (2005) and by Snyder (2019) were followed for this integrative literature review.

\subsection{Categorization of Modeling Papers Related to Substitution Demand Pattern}

The research on substitution demand modelling dates back to the early 1970s. Mosenson, a PhD student at MIT, in the $\mathrm{PhD}$ thesis submitted by him in 1970, proposed the solution to the product substitution problem. Mosenson and Dror (1972) came up with the possible patterns of qualitative substitution and complementarity among different goods. They defined substitution patterns as the system of 
substitution relationships among $\left(\begin{array}{l}n \\ 2\end{array}\right)$ in the context of consumer demand. In this study, the word cloud has been used to identify the major research topic in the inventory literature and to underline the most used words in the cloud. Figure1 shows the word cloud drawn based on the keywords in the research articles. Since the models on demand substitution were the key agenda of our review, the word cloud captured these words as the prominent key terms in these papers. The upcoming subsections are going to discuss some prominent articles that appeared in inventory literature based on the keywords as found in figure1.

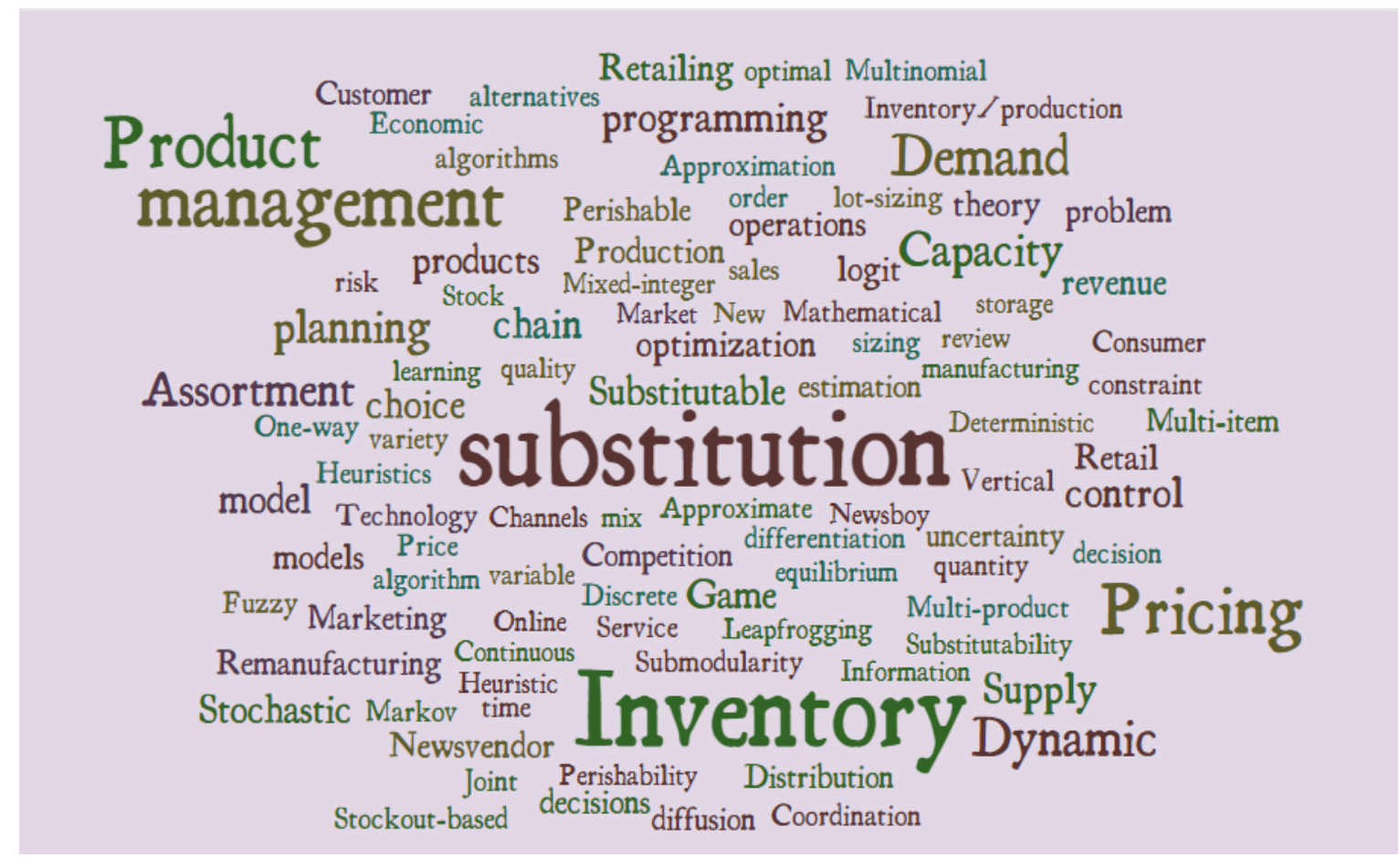

Figure 1 Word cloud formed from the keywords and abstracts of the research articles

\subsubsection{Articles related to assortment optimization}

The early papers in the 1970s (Pentico, 1974 and Pentico, 1976) focussed on assortment optimization where the seller deals in limited product variety owing to the resource constraints. Pentico (1988) extended the assortment-based substitution to two factors by taking into consideration the length as well as the strength of the steel beams while studying their substitutability. The strategy of assortment optimization is used by many retailers (Quelch \& Kenny, 1994). Rationalizing the range of assortment helps retailer optimize the costs and profits (Smith \& Agrawal, 2000). The cost reductions with the rationalization results from the fact that demand volumes for each of the variants increase with a smaller range. While the papers written in the twentieth century considered the identicalness of prices and cost structures among the substitutable products for the sake of modelling simplicity, the latter set of papers in the current century have considered the case of un-identical costs and prices also (Li et al. 2007). Huddiniah et al. (2018) linked the product variety with the increasing supply chain sophistication and proposed the framework of facilitating it with information technology. Ishichi et al. (2019) performed assortment planning and shelf space planning for substitutable products with the use of demand learning.

\subsubsection{Articles Related to Choice Models}

When the choice models are being talked about, the papers also differ in the variety. While some of the papers have considered the static choice modelling similar to the one by Gaur \& Honhon, 2006, the many others have considered dynamic choice modelling similar to the one by Yucel et al. 2009). While some papers have assumed the location choice models in which the customers substitute the demand between the neighbouring variants ( $\mathrm{Li}, 2007)$, many of the papers have assumed multinomial choice logit models (Suh \& Aydin, 2011; Aouad et al. 2018), with Hopp \& Xu (2005) considering the Bayesian Logit Model. Many of the research articles have taken the decision choice to be a Markov chains phenomenon (Bayinder et al. 2005; Yu et al. 2017; Desir et al. 2020). While Lin \& Sibdari (2009) deployed discrete choice model, Etebari (2020) used the nested logit model to capture the customer's choice process rather than the multinomial logit model considering that the latter one suffers from the independence of irrelevant alternatives limitation. The nature of demand pattern considered in the literature also spans from deterministic (Gurnani \& Drezner, 2000; Lang \& Domschke, 2010) to probabilistic (Pentico, 1974; Pasternack \& Drezner, 1991; Kraeselburd et al. 2004; Rao \& Swaminathan, 2004) and from Poisson distribution (Yadavalli et al. 2006; Xu et al. 2010; Burnetas \& Kanavetas, 2018) governed to innovation diffusion governed (Chanda, 2011; Chanda \& Agrawal, 2014; Chanda \& Das, 2015).

\subsubsection{Articles Related to Inventory Optimization for Substitutable Products}

Coming to inventory optimization for substitutable products, McGullivary and Silver (1978) was the first one to consider the demand substitution arising due to the stock-out of the referred product. Drezner et al. (1995) extended the 
EOQ Model of Harris to incorporate the effect of product substitution caused by the stock-out of the preferred product. Khouja et al. (1996) came up with the first version of the newsboy model for two items with substitutable demand. Bassok et al. (1999) said that product substitution offers the vendors a chance to pool their inventories and achieve lower inventory costs, which was later reinforced by $\mathrm{Hsu}$ et al. (2005). While the studies in the 1990s (Pasternack \& Drezner, 1991; Hsu \& Bassok, 1999) started with two substitutable products, the later studies (Rao et al. 2004; Shah \& Avittathur, 2007; Huang et al. (2011) have been extended to a set of $\mathrm{n}$ substitutable products, where $\mathrm{n}$ is any positive integer. Also, there have been many joint models on pricing and inventory in the twenty-first-century research such as Hopp et al. (2005); Maddah \& Bish (2007); Karakul and Chan; 2008); Akan et al. (2013); Yu et al. (2017) and many others. Within inventory optimization on substitutable products has also covered multiple perspectives such as joint replenishment (Yadavalli et al. 2006), information diffusion (Ganesh et al. 2008), supply chain coordination (Kraiselburd et al. 2004), re-manufacturing (Li et al. 2006; Bayinder et al. 2007). Shah et al. (2019) considered the case for the substitutable products where the demand is a quadratic function of time rather than a linear function.

\subsubsection{Articles Related to Inventory Optimization for Technological Substitutable Products}

Looking at the literature that talks of technological substitution, Chanda (2011) proposed the optimal price and quality setting model to determine the optimal price and quality for two substitutable generations of technology products. Chanda and Agarwal (2014) came up with the inventory optimization model for the two substitutable technology product generations. Chanda and Das (2015) discussed the dynamics of how technology generations get diffused in the market. Some of the prominent earlier works in the area of multi-generation substitution are Bass (1969), Norton \& Bass (1987), Islam \& Meade (1997), Kim et al. (2000), Danaher et al. (2001), Chanda \& Bardhan (2008), and Jiang \& Jain (2012).

\section{ELABORATE DESCRIPTION OF WORK IN THE LAST TWO DECADES}

After having been introduced to the variety of work in terms of assortment optimization, inventory optimization, pricing optimization, choice models and technological substitution, let us have a look at some of the papers in chronological sequence published in past twenty years. To begin with, the problem related to the approximation of the demand rates and demand substitution rates in case of stockout based substitution between multiple products was solved through the methods proposed by Anupindi et al. (1998). Extending the concept of product substitution in the manufacturing context, Balakrishnan and Geunes (2000) examined how flexible bill of materials with substitutable components and sub-assemblies can help reduce the inventories. Smith and Agrawal (2000) modelled the stochastic demand for substitutable items and an inventory optimization approach for profit maximization under capacity limitations. Rajaram and Tang (2001) extended the basic version of the newsvendor model to a scenario where an item with extra inventory can substitute the demand for an understocked item. Inderfurth (2004) and Xu et al. (2011) developed the optimal production model for the firms that are into manufacturing of new products as well as refurbishing of used product, both being substitutable. Rao et al. (2004) considered a one-way downward substitution for stochastic demand in multi-item inventory problem. Li et al. (2006) developed an optimal production planning problem for multiple products with demand substitution and remanufacturing of returned products.

Kok and Fisher (2007) proposed an approach to modelling the assortment decisions in which the customer may buy a substitute in the event of unavailability of his/her favourite product. Chen and Plambeck (2008) proved that inventory levels can be reduced by incorporating the learnings of substitution probabilities in the demand model. $\mathrm{Li}$ and $\mathrm{Ha}$ (2008) studied how the reactive capacity can help reduce the deviation between the supply and uncertain demand in case of substitutable products. Hopp and $\mathrm{Xu}$ (2008) studied how the interdependent decisions of assortment, inventory and price under demand substitution can be optimized by approximating the demand substitution behaviour. Shumsky and Zhang (2009) worked upon the optimal capacity allocation policy when multiple products correspond to multiple demand classes and customers can upgrade to higher demand class in the event of capacity depletion of their original demand class. Yucel et al. (2009) did the research work on optimal assortments in customerdriven demand substitution considering the practical issues related to supplier selection, product quality and shelf space limitations.

Bish et al. (2009) while exploring the case of a monopolist manufacturer of two products with substitutable demand under flexible capacity showed how the optimal capacity decision gets influenced by key demand parameters such as market size, market risk and nature of uncertainty. Tang and Yin (2009) developed a model for the joint determination of lot size and selling price under fixed as well as variable pricing strategy for two products with substitutable demand. Dawande et al. (2010) said that the production decisions in case of substitutable products are dependent upon the trade-off between the changeover costs and substitution costs. Gurler and Yilmaz (2010) considered a supply chain relationship between a retailer and manufacturer for two substitutable products under the buyback provision of the unsold inventory. Xu et al. (2010) worked upon optimal replenishment norms of substitutable products when the demand follows the Poisson distribution which is non-stationary by nature. Dutta and Chakraborty (2010) studied single period inventory optimization problem for two items with unilateral substitution in the imprecise business environment.

Goyal and Netessine (2011) said that demand uncertainty in case of substitutable products can be better mitigated by product flexibility rather than volume flexibility in the supply chain processes. Huang et al. (2011) studied the extension of the newsvendor problem with multiple products and partial product substitution. Amini and Li (2011) developed the integrated production planning and sales planning model when the new products are getting diffused in the market and substituting the earlier products. Honhon 
et al. (2011) deployed dynamic programming to optimize the inventory and assortment under stock-based substitution. Suh and Aydin (2011) formulated and proposed the solution to the price-setting problem for substitutable products whose demand is governed by a multinomial logit choice model influenced by the product price. Kim and Bell (2011) studied the influence of price-driven substitution on a firm's pricing decisions when it sells to multiple customer segments.

Akan et al. (2013) discussed how the manufacturer's ability to synchronise the product returns with the sales of a refurbished product can help optimize the profit in case of substitution between a new product and a refurbished product. Sainathan (2013) considered the demand substitution between competing perishable product variants where the product in the initial period of its shelf life has a higher perceived quality than the one in the later period of its shelf life. Saure and Zeevi (2013) showed how a retailer can derive useful insights on the consumer preferences by offering diverse types of assortments and observing the consumer's reactions, and incorporate that learning into his assortment planning exercise. Although Ganesh et al. (2014) said that the substitution reduces the need for information sharing by pooling the inventories. However, that is valid only for functional products and not for technology gadgets. Fisher and Vaidyanatham (2014) also developed a demand estimation method for substitutable products in retail assortments. Newman et al. (2014) examined how choicebased models can be estimated using the sales data in case of multiple substitutable products being sold by a single firm. Krommyda et al. (2015) optimized the order quantities for two substitutable products with stock dependent demand.

Wei and Zhao (2016) studied pricing for substitutable products under fuzzy environment, Zeppetella et al. (2017) considered the demand substitution under make-to-stock environment under capacity and production constraints; and optimized the production schedule. Pan et al. (2018) developed a stock-based substitution model for two products. Shlapp and Fleishmann (2018) derived the optimal inventory policy for a firm selling multiple products that are partially substitutable under capacity constraints. Chen et al. (2015) and; Chen and Cao (2020) showed how the information on substitution rates and primary demand rates can be learnt from the sales data on the fly. Dong et al. (2020) developed optimal pricing strategies for maximizing the expected profit.

\section{MULTI-CRITERIA \\ CATEGORIZATION OF WORK ON THE PARAMETERS OF THE RESEARCH OBJECTIVE AND SUBSTITUTION CHARACTERISTICS}

In this section, the existing literature has been classified thoroughly as per multiple criteria, such as the objective of the research, the propelling force behind the substitution, nature of substitution, the direction of substitution, etc. Table 1 captures the objective (viz. assortment optimization, inventory optimization, price optimization, demand estimation and capacity optimization), the nature of demand substitution (viz. assortment based, inventory-based, pricebased or technology-based), the propelling factor behind the substitution (viz. customer or vendor) and the direction of demand substitution (unidirectional or bi-directional), and the type of model used (viz. profit maximization or revenue maximization or utility maximization or cost minimization).

It can be observed that most of the research has been done on stockout based substitution, with the objective of inventory optimization for maximum profit. Although, many of the research papers have considered the other types of objectives and substitution scenarios.

Table 1 Analysis of existing literature on modelling for substitutable products in terms of objective, nature of substitution and model used

\begin{tabular}{|c|c|c|c|c|c|c|c|c|c|c|c|c|c|c|c|c|c|}
\hline \multirow{2}{*}{ Work } & \multicolumn{5}{|c|}{ Study Objective } & \multicolumn{4}{|c|}{$\begin{array}{c}\text { Nature of } \\
\text { substitution }\end{array}$} & \multicolumn{2}{|c|}{$\begin{array}{l}\text { Propelling } \\
\text { Force }\end{array}$} & \multicolumn{2}{|c|}{$\begin{array}{c}\text { Directio } \\
\mathbf{n}\end{array}$} & \multicolumn{4}{|c|}{ Optimization Model } \\
\hline & $A$ & I & $\mathbf{P}$ & C & D & $A B$ & $\mathbf{S}$ & $\mathbf{P}$ & $\mathbf{T}$ & C & V & U & B & $\begin{array}{l}P \\
M\end{array}$ & $\begin{array}{l}\mathrm{R} \\
\mathrm{M}\end{array}$ & $\begin{array}{l}U \\
M\end{array}$ & $\begin{array}{l}C \\
M\end{array}$ \\
\hline Pentico (1974) & $\checkmark$ & $x$ & $x$ & $x$ & $x$ & $\checkmark$ & $x$ & $x$ & $x$ & $x$ & $\checkmark$ & $\checkmark$ & $x$ & $x$ & $x$ & $x$ & $\checkmark$ \\
\hline Pentico (1976) & $\checkmark$ & $x$ & $x$ & $x$ & $x$ & $\checkmark$ & $x$ & $x$ & $x$ & $x$ & $\checkmark$ & $\checkmark$ & $x$ & $x$ & $x$ & $x$ & $\checkmark$ \\
\hline McGillivray and Silver (1978) & $x$ & $\checkmark$ & $x$ & $x$ & $x$ & $x$ & $\checkmark$ & $x$ & $x$ & $x$ & $\checkmark$ & $x$ & $\checkmark$ & $x$ & $x$ & $x$ & $\checkmark$ \\
\hline McGuire and Staelin (1983) & $x$ & $x$ & $\checkmark$ & $x$ & $x$ & $x$ & $x$ & $\checkmark$ & $x$ & $x$ & $\checkmark$ & $\checkmark$ & $x$ & $\checkmark$ & $x$ & $x$ & $x$ \\
\hline Norton and Bass (1987) & $x$ & $\checkmark$ & $x$ & $x$ & $x$ & $x$ & $x$ & $x$ & $\checkmark$ & $\checkmark$ & $x$ & $\checkmark$ & $x$ & $x$ & $\checkmark$ & $x$ & $x$ \\
\hline Parlar (1988) & $x$ & $\checkmark$ & $x$ & $x$ & $x$ & $x$ & $\checkmark$ & $x$ & $x$ & $\checkmark$ & $x$ & $x$ & $\checkmark$ & $\checkmark$ & $x$ & $x$ & $x$ \\
\hline Pentico (1988) & $\checkmark$ & $x$ & $x$ & $x$ & $x$ & $\checkmark$ & $x$ & $x$ & $x$ & $x$ & $\checkmark$ & $\checkmark$ & $x$ & $x$ & $x$ & $x$ & $\checkmark$ \\
\hline $\begin{array}{l}\text { Pasternack and Drezner } \\
\text { (1991) }\end{array}$ & $x$ & $\checkmark$ & $x$ & $x$ & $x$ & $x$ & $\checkmark$ & $x$ & $x$ & $x$ & $\checkmark$ & $x$ & $\checkmark$ & $\checkmark$ & $x$ & $x$ & $x$ \\
\hline Bitran \& Dasu (1992) & $x$ & $\checkmark$ & $x$ & $x$ & $x$ & $x$ & $\checkmark$ & $x$ & $x$ & $x$ & $\checkmark$ & $\checkmark$ & $x$ & $\checkmark$ & $x$ & $x$ & $x$ \\
\hline
\end{tabular}


Nagpal et al.: The First Half-Century of Decision Modelling for Substitutable Products: A Literature Review and Bibliographic Analysis 266

Operations and Supply Chain Management 14(3) pp. $261-276$ C 2021

Table 1 Analysis of existing literature on modelling for substitutable products in terms of objective, nature of substitution and model used (Con't)

\begin{tabular}{|c|c|c|c|c|c|c|c|c|c|c|c|c|c|c|c|c|c|}
\hline \multirow{2}{*}{ Work } & \multicolumn{5}{|c|}{ Study Objective } & \multicolumn{4}{|c|}{$\begin{array}{c}\text { Nature of } \\
\text { substitution }\end{array}$} & \multicolumn{2}{|c|}{$\begin{array}{l}\text { Propelling } \\
\text { Force }\end{array}$} & \multicolumn{2}{|c|}{$\begin{array}{c}\text { Directio } \\
n\end{array}$} & \multicolumn{4}{|c|}{ Optimization Model } \\
\hline & A & I & $\mathbf{P}$ & C & D & $A B$ & $\mathbf{S}$ & $\mathbf{P}$ & $\mathrm{T}$ & C & $\mathbf{V}$ & U & B & $\begin{array}{l}P \\
M\end{array}$ & $\begin{array}{l}\mathbf{R} \\
\mathrm{M}\end{array}$ & $\begin{array}{l}\mathrm{U} \\
\mathrm{M}\end{array}$ & $\begin{array}{l}\mathrm{C} \\
\mathrm{M}\end{array}$ \\
\hline Bitran and Gilbert (1994) & $x$ & $\checkmark$ & $x$ & $x$ & $x$ & $x$ & $\checkmark$ & $x$ & $x$ & $x$ & $\checkmark$ & $\checkmark$ & $x$ & $x$ & $x$ & $x$ & $\checkmark$ \\
\hline Chand et al. (1994) & $\checkmark$ & $\checkmark$ & $x$ & $x$ & $x$ & $\checkmark$ & $x$ & $x$ & $x$ & $\checkmark$ & $x$ & $\checkmark$ & $x$ & $x$ & $x$ & $x$ & $\checkmark$ \\
\hline Drezner et al. (1995) & $x$ & $\checkmark$ & $x$ & $x$ & $x$ & $x$ & $\checkmark$ & $x$ & $x$ & $x$ & $\checkmark$ & $x$ & $\checkmark$ & $x$ & $x$ & $x$ & $\checkmark$ \\
\hline Khouja et al. (1996) & $x$ & $\checkmark$ & $x$ & $x$ & $x$ & $x$ & $\checkmark$ & $x$ & $x$ & $\checkmark$ & $x$ & $x$ & $\checkmark$ & $\checkmark$ & $x$ & $x$ & $x$ \\
\hline $\begin{array}{l}\text { Lippman and McCardle } \\
\text { (1997) }\end{array}$ & $x$ & $\checkmark$ & $x$ & $x$ & $x$ & $x$ & $\checkmark$ & $x$ & $x$ & $\checkmark$ & $x$ & $x$ & $\checkmark$ & $\checkmark$ & $x$ & $x$ & $x$ \\
\hline Birge et al. (1998) & $x$ & $x$ & $\checkmark$ & $\checkmark$ & $\checkmark$ & $x$ & $x$ & $\checkmark$ & $x$ & $\checkmark$ & $x$ & $x$ & $\checkmark$ & $\checkmark$ & $x$ & $x$ & $x$ \\
\hline Bassok et al. (1999) & $x$ & $\checkmark$ & $x$ & $x$ & $x$ & $x$ & $\checkmark$ & $x$ & $x$ & $x$ & $\checkmark$ & $\checkmark$ & $x$ & $\checkmark$ & $x$ & $x$ & $x$ \\
\hline Ernst and Kouvelis (1999) & $x$ & $\checkmark$ & $x$ & $x$ & $x$ & $x$ & $\checkmark$ & $x$ & $x$ & $\checkmark$ & $x$ & $x$ & $\checkmark$ & $\checkmark$ & $x$ & $x$ & $x$ \\
\hline Hsu and Bassok (1999) & $x$ & $\checkmark$ & $x$ & $x$ & $x$ & $x$ & $\checkmark$ & $x$ & $x$ & $x$ & $\checkmark$ & $\checkmark$ & $x$ & $\checkmark$ & $x$ & $x$ & $x$ \\
\hline Van Ryzin \& Mahajan (1999) & $\checkmark$ & $\checkmark$ & $x$ & $x$ & $x$ & $\checkmark$ & $x$ & $x$ & $x$ & $\checkmark$ & $x$ & $x$ & $\checkmark$ & $\checkmark$ & $x$ & $x$ & $x$ \\
\hline $\begin{array}{l}\text { Balakrishnan and Geunes } \\
(2000)\end{array}$ & $x$ & $\checkmark$ & $x$ & $x$ & $x$ & $x$ & $\checkmark$ & $x$ & $x$ & $x$ & $\checkmark$ & $\checkmark$ & $x$ & $x$ & $x$ & $x$ & $\checkmark$ \\
\hline Duenyas and Tsai (2000) & $x$ & $\checkmark$ & $x$ & $x$ & $x$ & $x$ & $\checkmark$ & $x$ & $x$ & $x$ & $\checkmark$ & $\checkmark$ & $x$ & $\checkmark$ & $x$ & $x$ & $x$ \\
\hline Gurnani and Drezner (2000) & $x$ & $\checkmark$ & $x$ & $x$ & $x$ & $x$ & $\checkmark$ & $x$ & $x$ & $x$ & $\checkmark$ & $\checkmark$ & $x$ & $\checkmark$ & $x$ & $x$ & $x$ \\
\hline Smith \& Agrawal (2000) & $\checkmark$ & $\checkmark$ & $x$ & $x$ & $x$ & $x$ & $\checkmark$ & $x$ & $x$ & $\checkmark$ & $x$ & $x$ & $\checkmark$ & $\checkmark$ & $x$ & $x$ & $x$ \\
\hline Rajaram \& Teng (2001) & $\checkmark$ & $\checkmark$ & $x$ & $x$ & $x$ & $x$ & $\checkmark$ & $x$ & $x$ & $x$ & $\checkmark$ & $x$ & $\checkmark$ & $\checkmark$ & $x$ & $x$ & $x$ \\
\hline $\begin{array}{l}\text { Mahajan and van Ryzin } \\
\text { (2001a) }\end{array}$ & $x$ & $\checkmark$ & $x$ & $x$ & $x$ & $x$ & $\checkmark$ & $x$ & $x$ & $\checkmark$ & $x$ & $x$ & $\checkmark$ & $\checkmark$ & $x$ & $x$ & $x$ \\
\hline $\begin{array}{l}\text { Mahajan and van Ryzin } \\
(2001 \mathrm{~b})\end{array}$ & $x$ & $\checkmark$ & $x$ & $x$ & $x$ & $x$ & $\checkmark$ & $x$ & $x$ & $\checkmark$ & $x$ & $x$ & $\checkmark$ & $\checkmark$ & $x$ & $x$ & $x$ \\
\hline Avsar and Gursoy (2002) & $x$ & $\checkmark$ & $x$ & $x$ & $x$ & $x$ & $\checkmark$ & $x$ & $x$ & $\checkmark$ & $x$ & $x$ & $\checkmark$ & $\checkmark$ & $x$ & $x$ & $x$ \\
\hline Agarwal \& Smith (2003) & $\checkmark$ & $x$ & $x$ & $x$ & $x$ & $\checkmark$ & $x$ & $x$ & $x$ & $\checkmark$ & $x$ & $x$ & $\checkmark$ & $\checkmark$ & $x$ & $x$ & $x$ \\
\hline Netessine and Rudi (2003) & $x$ & $\checkmark$ & $x$ & $x$ & $x$ & $x$ & $\checkmark$ & $x$ & $x$ & $\checkmark$ & $x$ & $x$ & $\checkmark$ & $\checkmark$ & $x$ & $x$ & $x$ \\
\hline Kraiselburd et al. (2004) & $x$ & $\checkmark$ & $x$ & $x$ & $x$ & $x$ & $\checkmark$ & $x$ & $x$ & $x$ & $\checkmark$ & $x$ & $\checkmark$ & $\checkmark$ & $x$ & $x$ & $x$ \\
\hline Rao et al. (2004) & $x$ & $\checkmark$ & $x$ & $x$ & $x$ & $x$ & $\checkmark$ & $x$ & $x$ & $x$ & $\checkmark$ & $\checkmark$ & $x$ & $x$ & $x$ & $x$ & $\checkmark$ \\
\hline Bayindir et al. (2005) & $x$ & $\checkmark$ & $x$ & $x$ & $x$ & $x$ & $\checkmark$ & $x$ & $x$ & $x$ & $\checkmark$ & $\checkmark$ & $x$ & $\checkmark$ & $x$ & $x$ & $x$ \\
\hline Boyaci (2005) & $x$ & $\checkmark$ & $x$ & $x$ & $x$ & $x$ & $\checkmark$ & $x$ & $x$ & $x$ & $\checkmark$ & $x$ & $\checkmark$ & $\checkmark$ & $x$ & $x$ & $x$ \\
\hline Cachon et al. (2005) & $\checkmark$ & $x$ & $x$ & $x$ & $x$ & $\checkmark$ & $x$ & $x$ & $x$ & $\checkmark$ & $x$ & $x$ & $\checkmark$ & $\checkmark$ & $x$ & $x$ & $x$ \\
\hline Hopp \& Xu (2005) & $\checkmark$ & $x$ & $\checkmark$ & $x$ & $x$ & $\checkmark$ & $x$ & $x$ & $x$ & $\checkmark$ & $x$ & $x$ & $\checkmark$ & $\checkmark$ & $x$ & $\checkmark$ & $x$ \\
\hline Hsu et al. (2005) & $x$ & $\checkmark$ & $x$ & $x$ & $x$ & $x$ & $\checkmark$ & $x$ & $x$ & $x$ & $\checkmark$ & $\checkmark$ & $x$ & $x$ & $x$ & $x$ & $\checkmark$ \\
\hline Gaur \& Honhon (2006) & $\checkmark$ & $\checkmark$ & $x$ & $x$ & $x$ & $\checkmark$ & $\checkmark$ & $x$ & $x$ & $\checkmark$ & $x$ & $x$ & $\checkmark$ & $\checkmark$ & $x$ & $x$ & $x$ \\
\hline Li et al. (2006) & $x$ & $\checkmark$ & $x$ & $x$ & $x$ & $x$ & $\checkmark$ & $x$ & $x$ & $x$ & $\checkmark$ & $\checkmark$ & $x$ & $x$ & $x$ & $x$ & $\checkmark$ \\
\hline Bayindir et al. (2007) & $x$ & $\checkmark$ & $x$ & $x$ & $x$ & $x$ & $\checkmark$ & $x$ & $x$ & $x$ & $\checkmark$ & $\checkmark$ & $x$ & $\checkmark$ & $x$ & $x$ & $x$ \\
\hline Cachon \& Kok (2007) & $\checkmark$ & $x$ & $\checkmark$ & $x$ & $x$ & $\checkmark$ & $x$ & $x$ & $x$ & $\checkmark$ & $x$ & $x$ & $\checkmark$ & $\checkmark$ & $x$ & $x$ & $x$ \\
\hline Kok \& Fisher (2007) & $\checkmark$ & $\checkmark$ & $x$ & $x$ & $\checkmark$ & $\checkmark$ & $\checkmark$ & $x$ & $x$ & $\checkmark$ & $x$ & $x$ & $\checkmark$ & $\checkmark$ & $x$ & $x$ & $x$ \\
\hline Li (2007) & $\checkmark$ & $\checkmark$ & $x$ & $x$ & $x$ & $\checkmark$ & $x$ & $x$ & $x$ & $\checkmark$ & $x$ & $x$ & $\checkmark$ & $\checkmark$ & $x$ & $x$ & $x$ \\
\hline Liu and Lee, (2007) & $x$ & $\checkmark$ & $x$ & $x$ & $x$ & $x$ & $\checkmark$ & $x$ & $x$ & $x$ & $\checkmark$ & $\checkmark$ & $x$ & $x$ & $x$ & $\checkmark$ & $x$ \\
\hline Maddah \& Bish (2007) & $\checkmark$ & $\checkmark$ & $\checkmark$ & $x$ & $x$ & $\checkmark$ & $x$ & $x$ & $x$ & $\checkmark$ & $x$ & $x$ & $\checkmark$ & $\checkmark$ & $x$ & $x$ & $x$ \\
\hline Serin (2007) & $x$ & $\checkmark$ & $x$ & $x$ & $x$ & $x$ & $\checkmark$ & $x$ & $x$ & $\checkmark$ & $x$ & $x$ & $\checkmark$ & $\checkmark$ & $x$ & $x$ & $x$ \\
\hline Shah and Avittathur (2007) & $x$ & $\checkmark$ & $x$ & $x$ & $x$ & $x$ & $\checkmark$ & $x$ & $x$ & $\checkmark$ & $x$ & $\checkmark$ & $x$ & $\checkmark$ & $x$ & $x$ & $x$ \\
\hline Tang and Yin (2007) & $x$ & $\checkmark$ & $\checkmark$ & $x$ & $x$ & $x$ & $x$ & $\checkmark$ & $x$ & $\checkmark$ & $x$ & $\checkmark$ & $x$ & $\checkmark$ & $x$ & $x$ & $x$ \\
\hline Wu et al. (2007) & $x$ & $\checkmark$ & $\checkmark$ & $x$ & $x$ & $x$ & $x$ & $\checkmark$ & $x$ & $x$ & $\checkmark$ & $\checkmark$ & $x$ & $\checkmark$ & $x$ & $x$ & $x$ \\
\hline
\end{tabular}


Nagpal et al.: The First Half-Century of Decision Modelling for Substitutable Products: A Literature Review and Bibliographic Analysis Operations and Supply Chain Management 14(3) pp. $261-276$ (C) 2021

Table 1 Analysis of existing literature on modelling for substitutable products in terms of objective, nature of substitution and model used (Con't)

\begin{tabular}{|c|c|c|c|c|c|c|c|c|c|c|c|c|c|c|c|c|c|}
\hline \multirow{2}{*}{ Work } & \multicolumn{5}{|c|}{ Study Objective } & \multicolumn{4}{|c|}{$\begin{array}{c}\text { Nature of } \\
\text { substitution }\end{array}$} & \multicolumn{2}{|c|}{$\begin{array}{l}\text { Propelling } \\
\text { Force }\end{array}$} & \multicolumn{2}{|c|}{$\begin{array}{c}\text { Directio } \\
\mathrm{n}\end{array}$} & \multicolumn{4}{|c|}{ Optimization Model } \\
\hline & A & I & $\mathbf{P}$ & C & D & $A B$ & S & $\mathbf{P}$ & $\mathbf{T}$ & C & V & U & B & $\begin{array}{l}P \\
M\end{array}$ & $\begin{array}{l}R \\
M\end{array}$ & $\begin{array}{l}U \\
M\end{array}$ & $\begin{array}{l}C \\
M\end{array}$ \\
\hline Aydin and Porteus (2008) & $x$ & $\checkmark$ & $\checkmark$ & $x$ & $x$ & $x$ & $x$ & $\checkmark$ & $x$ & $x$ & $\checkmark$ & $\checkmark$ & $x$ & $\checkmark$ & $x$ & $x$ & $x$ \\
\hline Ganesh et al. (2008) & $x$ & $\checkmark$ & $x$ & $x$ & $x$ & $x$ & $\checkmark$ & $x$ & $x$ & $\checkmark$ & $x$ & $x$ & $\checkmark$ & $\checkmark$ & $x$ & $x$ & $x$ \\
\hline Hopp and Xu (2008) & $x$ & $\checkmark$ & $\checkmark$ & $x$ & $x$ & $x$ & $\checkmark$ & $x$ & $x$ & $x$ & $\checkmark$ & $x$ & $\checkmark$ & $\checkmark$ & $x$ & $x$ & $x$ \\
\hline Karakul (2008) & $x$ & $\checkmark$ & $\checkmark$ & $x$ & $x$ & $x$ & $x$ & $\checkmark$ & $x$ & $x$ & $\checkmark$ & $\checkmark$ & $x$ & $\checkmark$ & $x$ & $x$ & $x$ \\
\hline Karakul and Chan (2008) & $x$ & $\checkmark$ & $\checkmark$ & $x$ & $x$ & $x$ & $x$ & $\checkmark$ & $x$ & $x$ & $\checkmark$ & $\checkmark$ & $x$ & $\checkmark$ & $x$ & $x$ & $x$ \\
\hline $\begin{array}{l}\text { Nagarajan and Rajagopalan } \\
(2008)\end{array}$ & $x$ & $\checkmark$ & $x$ & $x$ & $x$ & $x$ & $\checkmark$ & $x$ & $x$ & $\checkmark$ & $x$ & $x$ & $\checkmark$ & $\checkmark$ & $x$ & $x$ & $x$ \\
\hline Sivakumar (2008) & $x$ & $\checkmark$ & $x$ & $x$ & $x$ & $x$ & $\checkmark$ & $x$ & $x$ & $x$ & $\checkmark$ & $x$ & $\checkmark$ & $x$ & $x$ & $x$ & $\checkmark$ \\
\hline Bish et al. (2009) & $x$ & $x$ & $x$ & $\checkmark$ & $\checkmark$ & $x$ & $x$ & $\checkmark$ & $x$ & $x$ & $\checkmark$ & $x$ & $\checkmark$ & $\checkmark$ & $x$ & $x$ & $x$ \\
\hline Dong et al. (2009) & $x$ & $x$ & $\checkmark$ & $x$ & $x$ & $x$ & $x$ & $\checkmark$ & $x$ & $\checkmark$ & $x$ & $\checkmark$ & $x$ & $x$ & $\checkmark$ & $x$ & $x$ \\
\hline Gurler, Oztop, and Sen (2009) & $x$ & $\checkmark$ & $\checkmark$ & $x$ & $x$ & $x$ & $\checkmark$ & $x$ & $x$ & $x$ & $\checkmark$ & $\checkmark$ & $x$ & $\checkmark$ & $x$ & $x$ & $x$ \\
\hline Hsieh and Wu (2009) & $x$ & $\checkmark$ & $\checkmark$ & $x$ & $x$ & $x$ & $x$ & $\checkmark$ & $x$ & $\checkmark$ & $x$ & $\checkmark$ & $x$ & $\checkmark$ & $x$ & $x$ & $x$ \\
\hline Vaagen et al. (2009) & $\checkmark$ & $\checkmark$ & $x$ & $x$ & $x$ & $x$ & $\checkmark$ & $x$ & $x$ & $\checkmark$ & $x$ & $x$ & $\checkmark$ & $\checkmark$ & $x$ & $x$ & $x$ \\
\hline Yang and Schrage (2009) & $x$ & $\checkmark$ & $x$ & $x$ & $x$ & $x$ & $\checkmark$ & $x$ & $x$ & $\checkmark$ & $x$ & $\checkmark$ & $x$ & $x$ & $x$ & $x$ & $\checkmark$ \\
\hline Yucel et al. (2009) & $\checkmark$ & $\checkmark$ & $x$ & $x$ & $x$ & $\checkmark$ & $\checkmark$ & $x$ & $x$ & $\checkmark$ & $x$ & $x$ & $\checkmark$ & $\checkmark$ & $x$ & $x$ & $x$ \\
\hline Akcay et al. (2010) & $x$ & $x$ & $\checkmark$ & $x$ & $x$ & $x$ & $x$ & $\checkmark$ & $x$ & $\checkmark$ & $x$ & $\checkmark$ & $x$ & $x$ & $\checkmark$ & $x$ & $x$ \\
\hline $\begin{array}{l}\text { Bish and Suwandechochai } \\
(2010)\end{array}$ & $x$ & $x$ & $x$ & $\checkmark$ & $\checkmark$ & $x$ & $x$ & $\checkmark$ & $x$ & $x$ & $\checkmark$ & $x$ & $\checkmark$ & $\checkmark$ & $x$ & $x$ & $x$ \\
\hline Chiang (2010) & $x$ & $\checkmark$ & $x$ & $x$ & $x$ & $x$ & $\checkmark$ & $x$ & $x$ & $\checkmark$ & $x$ & $x$ & $\checkmark$ & $\checkmark$ & $x$ & $x$ & $x$ \\
\hline Dawande et al. (2010) & $x$ & $\checkmark$ & $x$ & $x$ & $x$ & $x$ & $\checkmark$ & $x$ & $x$ & $x$ & $\checkmark$ & $\checkmark$ & $x$ & $x$ & $x$ & $x$ & $\checkmark$ \\
\hline Deniz et al. (2010) & $x$ & $\checkmark$ & $x$ & $x$ & $x$ & $x$ & $\checkmark$ & $x$ & $x$ & $\checkmark$ & $x$ & $\checkmark$ & $\checkmark$ & $\checkmark$ & $x$ & $x$ & $x$ \\
\hline Dutta and Chakraborty (2010) & $x$ & $\checkmark$ & $x$ & $x$ & $x$ & $x$ & $\checkmark$ & $x$ & $x$ & $x$ & $\checkmark$ & $\checkmark$ & $x$ & $\checkmark$ & $x$ & $x$ & $x$ \\
\hline Fadiloglu et al. (2010) & $\checkmark$ & $x$ & $x$ & $x$ & $x$ & $\checkmark$ & $x$ & $x$ & $x$ & $\checkmark$ & $x$ & $x$ & $\checkmark$ & $\checkmark$ & $x$ & $x$ & $x$ \\
\hline Gurler \& Yilmaz (2010) & $x$ & $\checkmark$ & $x$ & $x$ & $x$ & $x$ & $\checkmark$ & $x$ & $x$ & $x$ & $\checkmark$ & $x$ & $\checkmark$ & $\checkmark$ & $x$ & $x$ & $x$ \\
\hline Honhon et al. (2010) & $\checkmark$ & $\checkmark$ & $x$ & $x$ & $x$ & $x$ & $\checkmark$ & $x$ & $x$ & $\checkmark$ & $x$ & $x$ & $\checkmark$ & $\checkmark$ & $x$ & $x$ & $x$ \\
\hline Karakul and Chan (2010) & $x$ & $\checkmark$ & $\checkmark$ & $x$ & $x$ & $x$ & $x$ & $\checkmark$ & $x$ & $x$ & $\checkmark$ & $\checkmark$ & $x$ & $\checkmark$ & $x$ & $x$ & $x$ \\
\hline Lang and Domschke (2010) & $x$ & $\checkmark$ & $x$ & $x$ & $x$ & $x$ & $\checkmark$ & $x$ & $x$ & $x$ & $\checkmark$ & $\checkmark$ & $x$ & $x$ & $x$ & $x$ & $\checkmark$ \\
\hline Pineyro \& Viera (2010) & $x$ & $\checkmark$ & $x$ & $x$ & $x$ & $x$ & $\checkmark$ & $x$ & $x$ & $x$ & $\checkmark$ & $\checkmark$ & $x$ & $x$ & $x$ & $x$ & $\checkmark$ \\
\hline Tang \& Yin (2010) & $\checkmark$ & $\checkmark$ & $\checkmark$ & $x$ & $x$ & $x$ & $x$ & $\checkmark$ & $x$ & $x$ & $\checkmark$ & $x$ & $\checkmark$ & $\checkmark$ & $x$ & $x$ & $x$ \\
\hline Xu, Yao, and Zheng (2010) & $x$ & $\checkmark$ & $x$ & $x$ & $x$ & $x$ & $\checkmark$ & $x$ & $x$ & $\checkmark$ & $\checkmark$ & $\checkmark$ & $x$ & $x$ & $\checkmark$ & $x$ & $x$ \\
\hline Chanda (2011) & $x$ & $x$ & $\checkmark$ & $x$ & $x$ & $x$ & $x$ & $\checkmark$ & $\checkmark$ & $\checkmark$ & $\checkmark$ & $\checkmark$ & $x$ & $\checkmark$ & $x$ & $x$ & $x$ \\
\hline Huang et al. (2011) & $x$ & $\checkmark$ & $x$ & $x$ & $x$ & $x$ & $\checkmark$ & $x$ & $x$ & $\checkmark$ & $x$ & $x$ & $\checkmark$ & $\checkmark$ & $x$ & $x$ & $x$ \\
\hline Stavrulaki (2011) & $x$ & $\checkmark$ & $x$ & $x$ & $x$ & $x$ & $\checkmark$ & $x$ & $x$ & $\checkmark$ & $x$ & $x$ & $\checkmark$ & $\checkmark$ & $x$ & $x$ & $x$ \\
\hline Xia (2011) & $x$ & $x$ & $\checkmark$ & $x$ & $x$ & $x$ & $x$ & $\checkmark$ & $x$ & $x$ & $\checkmark$ & $\checkmark$ & $x$ & $\checkmark$ & $x$ & $x$ & $x$ \\
\hline Burkart et al. (2012) & $x$ & $x$ & $\checkmark$ & $x$ & $x$ & $x$ & $x$ & $\checkmark$ & $x$ & $\checkmark$ & $x$ & $x$ & $\checkmark$ & $x$ & $\checkmark$ & $x$ & $x$ \\
\hline Pan \& Honhon (2012) & $\checkmark$ & $x$ & $\checkmark$ & $x$ & $x$ & $\checkmark$ & $x$ & $x$ & $x$ & $\checkmark$ & $x$ & $x$ & $\checkmark$ & $\checkmark$ & $x$ & $x$ & $x$ \\
\hline Akan et al. (2013) & $x$ & $\checkmark$ & $\checkmark$ & $x$ & $x$ & $x$ & $x$ & $\checkmark$ & $x$ & $x$ & $\checkmark$ & $\checkmark$ & $x$ & $\checkmark$ & $x$ & $x$ & $x$ \\
\hline $\begin{array}{l}\text { Deflem and Nieuwenhuyse } \\
(2013)\end{array}$ & $x$ & $\checkmark$ & $x$ & $x$ & $x$ & $x$ & $\checkmark$ & $x$ & $x$ & $x$ & $\checkmark$ & $\checkmark$ & $x$ & $x$ & $x$ & $x$ & $\checkmark$ \\
\hline Gilland and Heese (2013) & $x$ & $\checkmark$ & $x$ & $x$ & $x$ & $x$ & $\checkmark$ & $x$ & $x$ & $\checkmark$ & $x$ & $x$ & $\checkmark$ & $\checkmark$ & $x$ & $x$ & $x$ \\
\hline Honhon \& Seshadri (2013) & $\checkmark$ & $x$ & $x$ & $x$ & $x$ & $\checkmark$ & $x$ & $x$ & $x$ & $\checkmark$ & $x$ & $x$ & $\checkmark$ & $\checkmark$ & $x$ & $x$ & $x$ \\
\hline Tan and Karabati (2013) & $x$ & $\checkmark$ & $x$ & $x$ & $x$ & $x$ & $\checkmark$ & $x$ & $x$ & $\checkmark$ & $x$ & $x$ & $\checkmark$ & $\checkmark$ & $x$ & $x$ & $x$ \\
\hline
\end{tabular}


Nagpal et al.: The First Half-Century of Decision Modelling for Substitutable Products: A Literature Review and Bibliographic Analysis 268

Operations and Supply Chain Management 14(3) pp. $261-276$ (C) 2021

Table 1 Analysis of existing literature on modelling for substitutable products in terms of objective, nature of substitution and model used (Con't)

\begin{tabular}{|c|c|c|c|c|c|c|c|c|c|c|c|c|c|c|c|c|c|}
\hline \multirow{2}{*}{ Work } & \multicolumn{5}{|c|}{ Study Objective } & \multicolumn{4}{|c|}{$\begin{array}{c}\text { Nature of } \\
\text { substitution }\end{array}$} & \multicolumn{2}{|c|}{$\begin{array}{l}\text { Propelling } \\
\text { Force }\end{array}$} & \multicolumn{2}{|c|}{$\begin{array}{c}\text { Directio } \\
\mathrm{n}\end{array}$} & \multicolumn{4}{|c|}{ Optimization Model } \\
\hline & A & 1 & $\mathbf{P}$ & C & D & $A B$ & $\mathbf{S}$ & $\mathbf{P}$ & $\mathbf{T}$ & C & V & $\mathbf{U}$ & B & $\begin{array}{l}P \\
M\end{array}$ & $\begin{array}{l}\mathbf{R} \\
\mathbf{M}\end{array}$ & $\begin{array}{l}U \\
M\end{array}$ & $\begin{array}{l}\text { C } \\
M\end{array}$ \\
\hline Chanda \& Aggarwal (2014) & $x$ & $\checkmark$ & $x$ & $x$ & $x$ & $x$ & $x$ & $x$ & $\checkmark$ & $\checkmark$ & $\checkmark$ & $\checkmark$ & $x$ & $x$ & $x$ & $x$ & $\checkmark$ \\
\hline Bernstein et al. (2015) & $x$ & $\checkmark$ & $x$ & $x$ & $x$ & $\checkmark$ & $\checkmark$ & $x$ & $x$ & $\checkmark$ & $x$ & $x$ & $\checkmark$ & $x$ & $\checkmark$ & $x$ & $x$ \\
\hline Chanda \& Das (2015) & $\checkmark$ & $x$ & $x$ & $x$ & $x$ & $x$ & $x$ & $x$ & $\checkmark$ & $\checkmark$ & $\checkmark$ & $\checkmark$ & $x$ & $\checkmark$ & $x$ & $x$ & $x$ \\
\hline Chen et al. (2015) & $x$ & $\checkmark$ & $x$ & $x$ & $x$ & $\checkmark$ & $x$ & $x$ & $x$ & $x$ & $\checkmark$ & $x$ & $\checkmark$ & $x$ & $x$ & $\checkmark$ & $x$ \\
\hline Cosgun et al. (2017) & $x$ & $x$ & $\checkmark$ & $x$ & $x$ & $x$ & $x$ & $\checkmark$ & $x$ & $x$ & $\checkmark$ & $x$ & $\checkmark$ & $\checkmark$ & $x$ & $x$ & $x$ \\
\hline Li \& Fu (2017) & $x$ & $\checkmark$ & $x$ & $x$ & $x$ & $x$ & $\checkmark$ & $x$ & $x$ & $\checkmark$ & $x$ & $x$ & $\checkmark$ & $\checkmark$ & $x$ & $x$ & $x$ \\
\hline $\begin{array}{l}\text { Mukhopadhyay \& Goswami } \\
(2017)\end{array}$ & $x$ & $\checkmark$ & $x$ & $x$ & $x$ & $x$ & $\checkmark$ & $x$ & $x$ & $\checkmark$ & $x$ & $\checkmark$ & $x$ & $x$ & $x$ & $x$ & $\checkmark$ \\
\hline Transchel (2017) & $x$ & $\checkmark$ & $x$ & $x$ & $x$ & $x$ & $\checkmark$ & $\checkmark$ & $x$ & $\checkmark$ & $x$ & $x$ & $\checkmark$ & $\checkmark$ & $x$ & $x$ & $x$ \\
\hline Yu et al. (2017) & $x$ & $\checkmark$ & $\checkmark$ & $x$ & $x$ & $\checkmark$ & $x$ & $\checkmark$ & $x$ & $x$ & $\checkmark$ & $\checkmark$ & $x$ & $\checkmark$ & $x$ & $x$ & $x$ \\
\hline Aouad et al. (2018) & $\checkmark$ & $\checkmark$ & $x$ & $x$ & $x$ & $\checkmark$ & $x$ & $x$ & $x$ & $\checkmark$ & $x$ & $x$ & $\checkmark$ & $\checkmark$ & $x$ & $x$ & $x$ \\
\hline Burnetas et al. (2018) & $x$ & $\checkmark$ & $x$ & $x$ & $x$ & $x$ & $\checkmark$ & $x$ & $x$ & $\checkmark$ & $x$ & $x$ & $\checkmark$ & $\checkmark$ & $x$ & $x$ & $x$ \\
\hline Ceryan et al. (2018) & $x$ & $x$ & $\checkmark$ & $x$ & $x$ & $x$ & $\checkmark$ & $\checkmark$ & $x$ & $\checkmark$ & $x$ & $x$ & $\checkmark$ & $\checkmark$ & $x$ & $x$ & $x$ \\
\hline Farahat \& Lee (2018) & $x$ & $\checkmark$ & $x$ & $x$ & $x$ & $\checkmark$ & $x$ & $x$ & $x$ & $\checkmark$ & $x$ & $x$ & $\checkmark$ & $\checkmark$ & $x$ & $x$ & $x$ \\
\hline Khademi \& Eksioglu (2018) & $x$ & $\checkmark$ & $x$ & $x$ & $x$ & $x$ & $\checkmark$ & $x$ & $x$ & $x$ & $\checkmark$ & $x$ & $\checkmark$ & $x$ & $x$ & $x$ & $\checkmark$ \\
\hline Schlapp \& Fleischmann (2018) & $x$ & $\checkmark$ & $x$ & $x$ & $x$ & $x$ & $\checkmark$ & $x$ & $x$ & $\checkmark$ & $x$ & $x$ & $\checkmark$ & $\checkmark$ & $x$ & $x$ & $x$ \\
\hline Surti et al. (2018) & $x$ & $\checkmark$ & $\checkmark$ & $x$ & $x$ & $x$ & $\checkmark$ & $\checkmark$ & $x$ & $\checkmark$ & $x$ & $x$ & $\checkmark$ & $\checkmark$ & $x$ & $x$ & $x$ \\
\hline Wan et al. (2018) & $\checkmark$ & $\checkmark$ & $x$ & $x$ & $\checkmark$ & $\checkmark$ & $x$ & $x$ & $x$ & $\checkmark$ & $x$ & $x$ & $\checkmark$ & $\checkmark$ & $x$ & $x$ & $x$ \\
\hline Zhang et al. (2018) & $x$ & $\checkmark$ & $x$ & $x$ & $x$ & $\checkmark$ & $x$ & $x$ & $x$ & $\checkmark$ & $x$ & $x$ & $\checkmark$ & $\checkmark$ & $x$ & $x$ & $x$ \\
\hline Aouad et al. (2019) & $\checkmark$ & $\checkmark$ & $x$ & $x$ & $x$ & $\checkmark$ & $x$ & $x$ & $x$ & $x$ & $\checkmark$ & $x$ & $\checkmark$ & $\checkmark$ & $x$ & $x$ & $x$ \\
\hline Feng et al. (2019) & $x$ & $\checkmark$ & $x$ & $x$ & $x$ & $x$ & $\checkmark$ & $x$ & $x$ & $\checkmark$ & $x$ & $\checkmark$ & $x$ & $x$ & $x$ & $x$ & $\checkmark$ \\
\hline Fu et al. (2019) & $x$ & $\checkmark$ & $x$ & $x$ & $x$ & $\checkmark$ & $\checkmark$ & $x$ & $x$ & $x$ & $\checkmark$ & $\checkmark$ & $x$ & $\checkmark$ & $x$ & $x$ & $x$ \\
\hline Geunes \& Su (2019) & $\checkmark$ & $\checkmark$ & $x$ & $x$ & $x$ & $\checkmark$ & $\checkmark$ & $x$ & $x$ & $\checkmark$ & $x$ & $x$ & $\checkmark$ & $\checkmark$ & $x$ & $x$ & $x$ \\
\hline Jing \& Mu (2019) & $x$ & $\checkmark$ & $x$ & $x$ & $x$ & $\checkmark$ & $x$ & $x$ & $x$ & $\checkmark$ & $x$ & $\checkmark$ & $x$ & $\checkmark$ & $x$ & $x$ & $x$ \\
\hline Kim \& Bell (2019) & $x$ & $\checkmark$ & $\checkmark$ & $x$ & $x$ & $x$ & $\checkmark$ & $\checkmark$ & $x$ & $\checkmark$ & $x$ & $x$ & $\checkmark$ & $\checkmark$ & $\checkmark$ & $x$ & $x$ \\
\hline Chan et al. (2020) & $\checkmark$ & $\checkmark$ & $x$ & $x$ & $x$ & $\checkmark$ & $\checkmark$ & $x$ & $x$ & $\checkmark$ & $\checkmark$ & $x$ & $\checkmark$ & $\checkmark$ & $x$ & $x$ & $x$ \\
\hline Jing \& Mu (2020) & $x$ & $\checkmark$ & $x$ & $x$ & $x$ & $\checkmark$ & $x$ & $x$ & $x$ & $x$ & $\checkmark$ & $\checkmark$ & $x$ & $\checkmark$ & $x$ & $x$ & $x$ \\
\hline Majumder et al. (2020) & $x$ & $\checkmark$ & $x$ & $x$ & $x$ & $\checkmark$ & $\checkmark$ & $x$ & $x$ & $\checkmark$ & $x$ & $x$ & $\checkmark$ & $\checkmark$ & $x$ & $x$ & $x$ \\
\hline Rasouli et al. (2020) & $x$ & $x$ & $\checkmark$ & $x$ & $x$ & $\checkmark$ & $x$ & $x$ & $x$ & $\checkmark$ & $x$ & $x$ & $\checkmark$ & $\checkmark$ & $x$ & $x$ & $x$ \\
\hline Hsieh \& Lai (2020) & $x$ & $\checkmark$ & $\checkmark$ & $x$ & $x$ & $\checkmark$ & $x$ & $x$ & $x$ & $\checkmark$ & $x$ & $\checkmark$ & $x$ & $\checkmark$ & $x$ & $x$ & $x$ \\
\hline $\begin{array}{l}\text { Antipov and Pokryshevskaya } \\
(2020)\end{array}$ & $\checkmark$ & $x$ & $x$ & $x$ & $\checkmark$ & $\checkmark$ & $x$ & $x$ & $x$ & $\checkmark$ & $x$ & $\checkmark$ & $x$ & $x$ & $x$ & $x$ & $x$ \\
\hline
\end{tabular}

Legends A: Assortment Optimization, I: Inventory Optimization, P: Price Optimization, C: Capacity Optimization, D: Demand Estimation, AB: Assortment Based, S: Stock-Based, P: Pricing-Based, T: Technology-based, C: Customer Influenced, VI: Vendor Influenced, U: Unidirectional, B: Bi-directional, PM: Profit Maximization, RM: Revenue Maximization, UM: Utility Maximization, CM: Cost Minimization 
In the next section, the key methods used for the optimization of inventories used in the research papers of the past five decades on modelling for substitutable demand have been elaborated.

\section{DISCUSSION ON THE METHODOLOGIES USED TO SOLVE OPTIMIZATION MODELS}

A variety of methods have been deployed to solve the optimization models, the most prominent of them being game theory, dynamic programming and newsvendor model. Shah and Avittathur (2007) developed the heuristics for solving the twin problem of the optimal assortment and optimal inventory in the retailing context under demand substitution and cannibalization. While studying the problem of substitution between a new product and remanufactured product, Pineyro and Viera (2010) found it to be NP-hard, and solved the same using tabu search. Rusmevichientong et al. (2010) and; Agrawal et al. (2016) worked on an online assortment optimization problem with a multinomial logit model based consumer choice model and dynamic demand learning by the retailers. Asad and Demirli (2010) developed MILP for optimal production scheduling under demand substitution in the steel rolling mills.
Zhao et al. (2012a and 2012b) analysed the pricesetting decision modelling of two substitutable products under imprecise manufacturing cost and customer demand using the game theory. Ma et al. (2015) extended the basic single product newsvendor model to a multi-product newsvendor model with demand substitution and developed computational algorithms for optimal assortment and optimal order quantities. Goyal et al. (2016) showed that assortment optimization modelling with dynamic substitutions under stochastic demand is NP-hard even it may be the simplest form of consumer choice model. Xu et al. (2016) developed inventory model for flexible substitution scheme in which the supplier has the flexibility to offer or not to offer the substitution and the customer has the flexibility to accept or reject it, and solved it using stochastic dynamic programming approach. Antipov and Pokryshevskaya (2020) examined the potential of machine learning methods- elastic nets, gradient boosting and random forests in identifying the complex substitution patterns from the POS data of the retailers.

Table 2 captures the solution approach used in the papers (viz. genetic algorithm, linear programming, game theory, newsboy model, nonlinear programming, mixed integer programming, stochastic programming, dynamic programming, etc.).

Table 2 The solution methodologies used in the research articles

\begin{tabular}{|l|l|}
\hline Method Deployed & Modelling studies in chronological order \\
\hline Markov & Duenyas and Tsai (2000); Bayindir et al. (2005); Sivakumar (2008); Yu et al. (2017); Desir et al. (2020) \\
\hline Logit choice model & Rusmevichientong et al. (2010); Agrawal et al. (2016); Aouad et al. (2018) \\
\hline Bayesian Logit Model & Hopp \& Xu (2005) \\
\hline Game Theory & $\begin{array}{l}\text { McGuire and Staelin (1983); Parlar (1988); Avsar and Gursoy (2002); Netessine and Rudi (2003); Boyaci } \\
\text { (2005); Serin (2007); Wu et al. (2007); Hsieh and Wu (2009); Chiang (2010); Tang \& Yin (2010); Xia (2011); } \\
\text { Hseih \& Lai (2020) }\end{array}$ \\
\hline $\begin{array}{l}\text { Pasternack \& Drezner (1991); Ernst and Kouvelis (1999); van Ryzin \& Mahajan (1999); Mahajan and van } \\
\text { Ryzin (2001a); Bayindir et al. (2007); Maddah \& Bish (2007); Serin (2007); Shah and Avittathur (2007); Wu } \\
\text { et al. (2007); Vaagen et al. (2009); Yang and Schrage (2009); Dutta and Chakraborty (2010); Gurler \& Yilmaz } \\
\text { (2010); Karakul and Chan (2010); Huang et al. (2011); Stavrulaki (2011); Deflem and Van Nieuwenhuyse } \\
\text { (2013); Maddah et al. (2014); Bernstein et al. (2015); Li \& Fu (2017); Surti et al. (2018) }\end{array}$ \\
\hline $\begin{array}{l}\text { Pentico (1974); Pentico (1976); Pentico (1988); Bitran and Gilbert (1994); Rao et al. (2004); Hsu et al. (2005); } \\
\text { Li et al. (2006); Nagarajan and Rajagopalan (2008); Dong et al. (2009); Akcay et al. (2010); Honhon et al. } \\
\text { (2010); Xu, Yao, and Zheng (2010); Wan et al. (2018); Fu et al. (2019) }\end{array}$ \\
\hline Dynamic Programming Shah \& Avittatathur (2007); Nagarajan \& Rajagopalan (2008); Deniz et al. (2009) \\
\hline Heuristics & $\begin{array}{l}\text { Bitran \& Dasu (1992); Netessine et al. (2002); Kraiselburd et al. (2004); Bish et al. (2009); Bish and } \\
\text { Suwandechochai (2010) }\end{array}$ \\
\hline Stochastic Programming & Agarwal \& Smith (2003) \\
\hline Linear Programming & Bassok et al. (1999) \\
\hline Greedy Search & $\begin{array}{l}\text { Balakrishnan and Geunes (2000); Gaur \& Honhon (2006); Lang and Domschke (2010); Pineyro \& Viera } \\
\text { (2010); Burkart et al. (2012) }\end{array}$ \\
\hline MILP & $\begin{array}{l}\text { Chand et al. (1994); Anupindi et al. (1998); Li (2007); Tang and Yin (2007); Honhon \& Seshadri (2013); } \\
\text { Chanda \& Aggarwal (2014); Chen et al. (2015) }\end{array}$ \\
\hline Non-Linear Programming
\end{tabular}




\section{KEY STATISTICS ON THE RESEARCH DONE TO DATE ON SUBSTITUTION MODELING}

This section discusses the key statistics of the existing research on modelling for substitutable demand. Figure 2 captures the fifteen most widely cited papers that have more than ten citations per year. The literature review by Khouja (1999) is the most widely cited paper, followed by Netessin \& Rudy (2003) and Kok \& Fisher (2007).

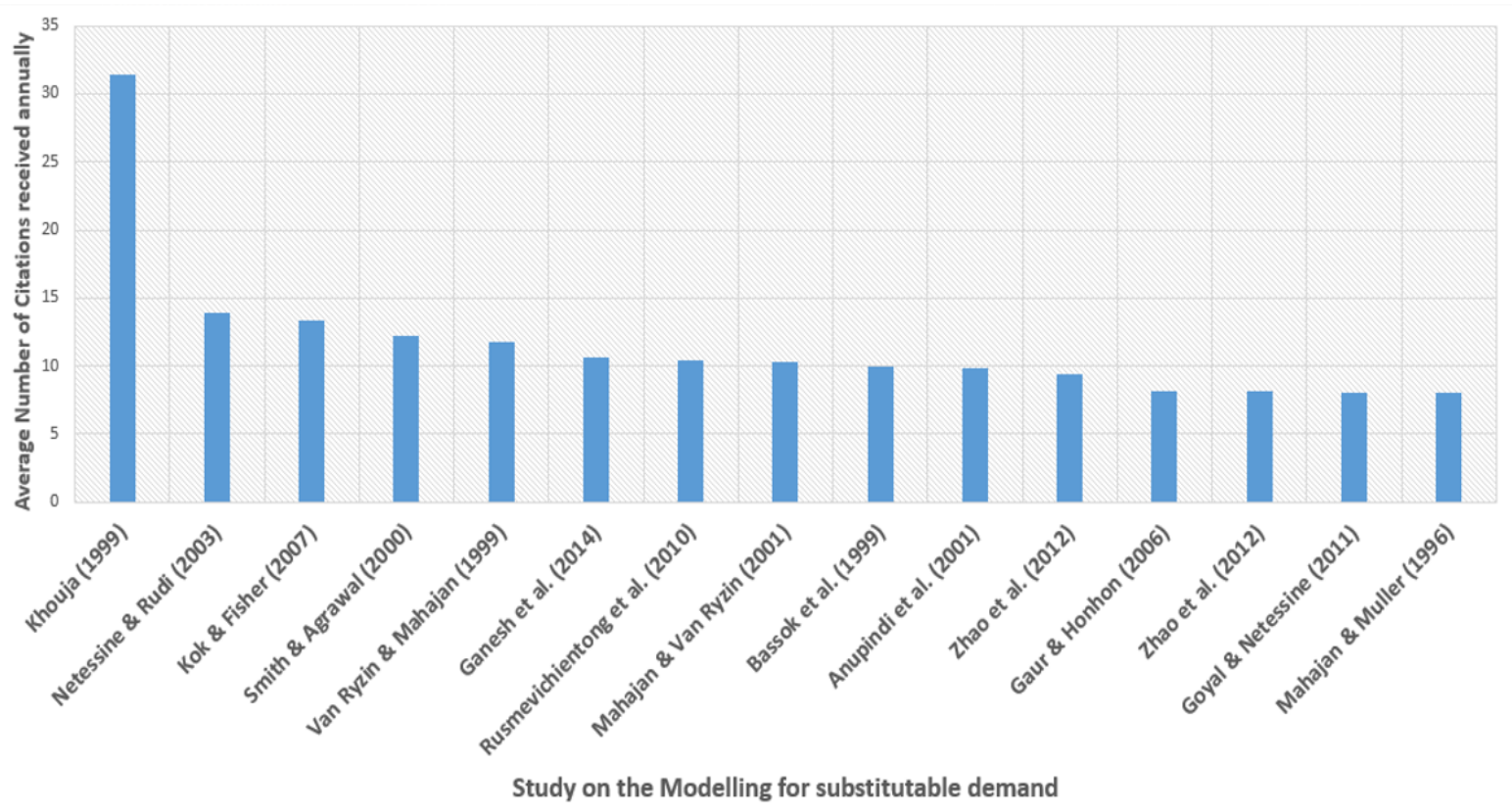

Figure 2 Papers with more than 8 citations per year since the year of publication

Figure 3 shows the number of research articles written around the theme of demand substitution over each of the periods of five-year length. It can be observed that the research interest in this domain has increased drastically over the two decades from 1990 till 2010 due to increasing substitutable product variety in every industry with the mass customization concepts becoming popular.

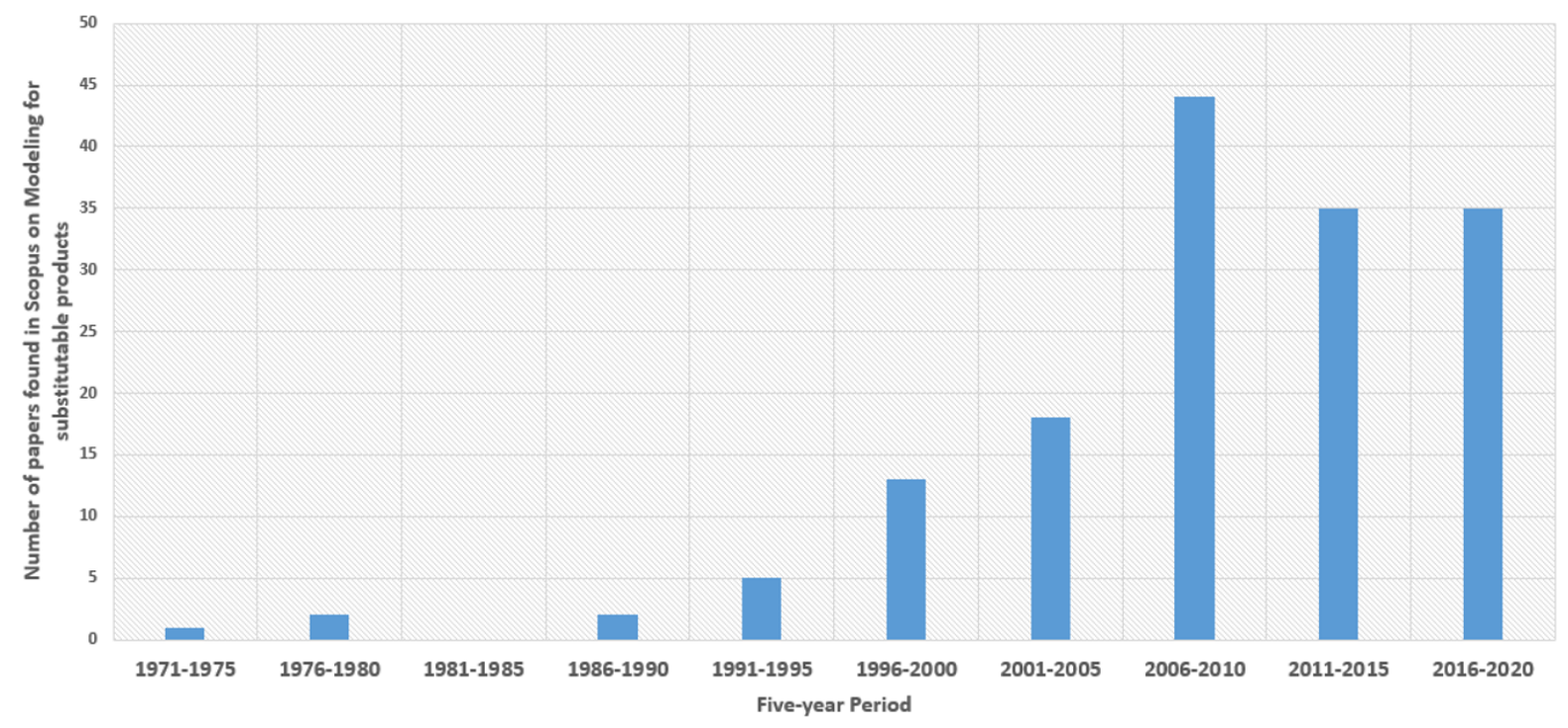

Figure 3 Trends in the five decades for research studies on demand substitution modelling

Figure 4 shows the most popular journals that have published the works on modelling for substitutable demand items. European Journal of Operational Research and
Operations Research are the two most popular journals for publishing the work in this domain. 


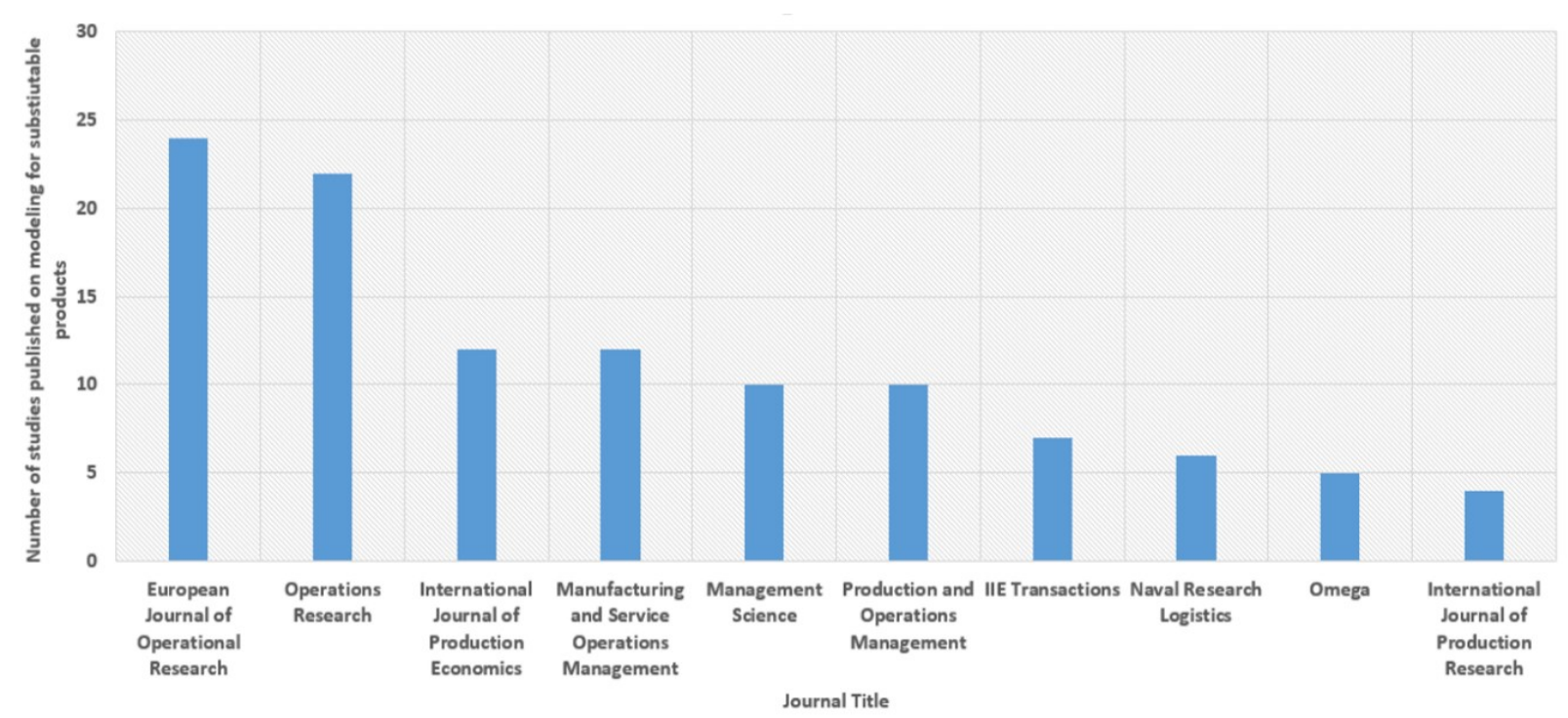

Figure 4 The number of studies published in the journals on modelling for substitutable products

In the next section, different methodologies used to solve the optimization models in the inventory literature have been discussed.

\section{ACHIEVEMENTS OF THE PAST WORK}

From this review, one can observe that the five decades have seen a lot of progress when one observes the research on the modelling for substitutable products. Irrespective of the nature of the substitution, the purpose of research in most of the articles is the maximization of the profit. Revenue maximization and Cost minimization are the other two models, while utility maximization or capacity maximization or planning horizon optimization are a few other models that have also been explored. Demand substitution carries both advantages as well as disadvantages for the firms. While the product substitution raises the level of demand uncertainty for each product variant, it also enables the firms to pool the risks and meet the demand of one product with the inventories of the other substitutable product.

The past work done on modelling for substitutable items is a good learning ground for the businesses in the realm of operations, supply chain management, business strategy, product management and marketing management. The papers written on demand modelling have given useful insights on the customer choice models and the purchase behaviour of the consumer. The studies to date have been able to cover a wide range of demand substitution models with diverse objectives and optimization approaches. This has been of good help to the industry practitioners and policymakers in making sound decisions related to assortment rationalization, pricing and inventory. The retail industry, in particular, has a lot to benefits to derive from these studies.

This review has been a decent attempt at collating all the existing research on the mentioned topic onto a common platform and classifying the same into broad categories. The review has also been a very exhaustive and comprehensive one and has touched upon multiple interdisciplinary perspectives of demand substitution. The review is expected to guide the academicians in disseminating the existing body of knowledge in the classroom related to demand substitution. It can be useful particularly in the teaching of subjects such as marketing, supply chain management, optimization and consumer behaviour. This review can be a point of reference for all the industry practitioners faced with the problem of managing items with substitutable demand. The review can also be of immense help to the researchers in finding out what has been done to date, and what needs to be done ahead.

\section{LIMITATIONS AND FUTURE DIRECTIONS}

Despite significant research progress in this domain, the work on the modelling for substitutable products can be extended further to bring it closer to the practical realities of the modern world business phenomenon. First, there needs to be more research on the consumer-driven locational choice models. Much of the existing research is on multinomial logit choice models that are not fit in the situations where the chance of choosing a product is independent of the chance of choosing another. Also, multinomial choice models are inappropriate when the product attributes are in the form of a continuous function. Second, even the uni-directional research in case of locational choice models is very rare while so many cases are known such as technological substitution, where the customer buys a model in the neighbouring range only, and will always upgrade to a higher version.

Third, most of the research assumes that customers make a rational choice, while it is known that cognitive biases have a strong influence on the choices made by the consumer. Apart from the cognitive biases, the customers are sometimes subjected to information asymmetry or peer pressure or time constraints, which lead them to the path of making irrational and non-stationary choices that cannot be specified by fixed preference relations. Therefore, there exists a need to have more of research that can entertain the unexpected choices of the consumer under the bounded rationality.

Fourth, while decisions related to assortment, inventory and price have received the attention, the work on capacity 
optimization has a much more scope to offer than what has been explored. The inadequate capacity at the manufacturer's end or the supplier's end can result in stockouts, and the allocation of the fixed capacity to the multiple substitutable products can be optimized within the constraints. Fifth, the areas of risk mitigation through the supply chain contracts and the three Cs of coordination, cooperation and collaboration may be explored for substitutable products. Sixth, the work on the inventory modelling for technology generations can be strengthened, given the fact that the World is witnessing the digital age where the conventional products are being transformed into intelligent and connected products.

Seventh, with the advent of business intelligence, a lot of data is available at the click of the mouse, and the real time decisions can be made on the basis of algorithms built in the computing systems. This has reduced the response time of the businesses to the changing environment, and therefore, can also have the implications for the type of decision models to be used. The research can happen on integrating the models with the latest development in information systems to leverage the effect of the computing revolution.

Eight, we have seen that much of the literature on decision modelling for substitutable products has considered only one objective at a time. While in the real world, we may have multiple conflicting goals that need to be optimized at the expense of one another. This would call for the goal programming or multi-criteria decision making. If future research can address this gap, that shall be useful.

Last but not the least, it has been observed quite often that the value of unit inventory changes (generally depreciates) with time, a fact that can be considered in the future papers on optimization for substitutable products.

\section{ACKNOWLEDGEMENTS}

The authors acknowledge the infrastructure support rendered by Birla Institute of Technology and Science, Pilani, Rajasthan, India for this research.

\section{REFERENCES}

Agrawal, N., \& Smith, S.A. (2003). Optimal retail assortments for substitutable items purchased in sets. Naval Research Logistics, 50(7), 793-822.

Akan, M., Ata, B. \& Savaskan-Ebert, R.C. (2013). Dynamic pricing of re-manufacturable products under demand substitution: a product life cycle model. Annals of Operations Research, 211(1), 1-25. https://doi.org/10.1007/s10479-0131409-1

Akcay, Y., Natarajan, H.P., \& Xu, S.H. (2010). Joint dynamic pricing of multiple perishable products under consumer choice. Management Science, 56(8), 1345-1361.

Amini, M. \& Li, H. (2011). Supply chain configuration for the diffusion of new products: An integrated optimization approach. Omega, 39(3), 313-322.

An, L., \& Ramachandran, B. (2005, July). System dynamics model to understand demand conditioning dynamics in supply chains. In The 23rd International Conference of the System Dynamics Society.

Anupindi, R., Dada, M. \& Gupta, S. (1998). Estimation of Consumer Demand with Stock-Out Based Substitution: An Application to Vending Machine Products. Marketing Science, 17(4), 406-423, https://doi.org/10.1287/mksc.17.4.406
Antipov, E.A., Pokryshevskaya, E.B. (2020). Interpretable machine learning for demand modeling with high-dimensiona data using Gradient Boosting Machines and Shapley values. Journal of Revenue and Pricing Management, 19(5), 355-364

Aouad A., Levi R., Segev D. (2018). Greedy-like algorithms for dynamic assortment planning under multinomial logit preferences. Operations Research, 66(5), 1321-1345.

Aouad A., Levi R., Segev D. (2019). Approximation algorithms for dynamic assortment optimization models. Mathematics of Operations Research, 44(2), 487-511.

Asad, R. \& Demirli, K. (2010). Production scheduling in steel rolling mills with demand substitution: Rolling horizon implementation and approximations. International Journal of Production Economics, 126(2), 361-369.

Avsar, Z.M., \& Gursoy, M.B. (2002). Inventory control under substitutable demand: A stochastic game application. Naval Research Logistics, 49(4), 359-375.

Aydin, G., \& Porteus, E.L. (2008). Joint inventory and pricing decisions for an assortment. Operations Research, 56(5), 1247-1255.

Balakrishnan, A. \& Geunes, J. (2000). Requirements planning with substitutions: exploiting bill-of-materials flexibility in production planning. Manufacturing and Service Operations Management, 2(2), 166-185

Bass, F. M. (1969). A new product growth model for consumer durables. Management Science, 50, 1825-1832.

Bassok, Y., Anupindi, R., \& Akella, R. (1999). Single-period multiproduct inventory models with substitution. Operations Research, 47(4), 632-642.

Bayindir, Z.P., Erkip, N., \& Gullu, R. (2005). Assessing the benefits of remanufacturing option under one-way substitution. The Journal of the Operational Research Society, 56(3), 286-296.

Bayindir, Z.P., Erkip, N., \& Gullu, R. (2007). Assessing the benefits of remanufacturing option under one-way substitution and capacity constraint. Computers \& Operations Research, 34(2), 487-514.

Bernstein F., Gurhan Kok A., Xie L. (2015). Dynamic assortment customization with limited inventories. Manufacturing and Service Operations Management, 17(4), 538-553.

Birge, J.R., Drogosz, J., \& Duenyas, I. (1998). Setting single-period optimal capacity levels and prices for substitutable products. International Journal of Flexible Manufacturing Systems, 10(4), 407-430.

Bish, E.K., \& Suwandechochai, R. (2010). Optimal capacity for substitutable products under operational postponement European Journal of Operational Research, 207(2), 775-783.

Bish, E.K., Liu, J., \& Suwandechochai, R. (2009). Optimal capacity, product substitution, linear demand models, and uncertainty. The Engineering Economist, 54(2), 109-151.

Bitran, G.R., \& Dasu, S. (1992). Ordering policies in an environment of stochastic yields and substitutable demands. Operations Research, 40(5), 999-1017.

Bitran, G.R., \& Gilbert, S.M. (1994). Co-production processes with random yields in the semiconductor industry. Operations Research, 42(3), 476-491.

Boyaci, T. (2005). Competitive stocking and coordination in a multiple-channel distribution system. IIE Transactions, 37(5), 407-427.

Burkart, W.R., Klein, R., \& Mayer, S. (2012). Product line pricing for services with capacity constraints and dynamic substitution. European Journal of Operational Research, 219(2), 347-359.

Burnetas A., Kanavetas O. (2018). Inventory policies for two products under Poisson demand: Interaction between demand substitution, limited storage capacity and replenishment time uncertainty. Naval Research Logistics, 65(8), 676-698.

Cachon, G. P., \& Kok, A. G. (2007). Category management and coordination in retail assortment planning in the presence of 
basket shopping consumers. Management Science, 53(6), 934951.

Cachon, G. P., Terwiesch, C., \& Xu, Y. (2005). Retail assortment planning in the presence of consumer search. Manufacturing \& Service Operations Management, 7(4), 330-346.

Ceryan O., Duenyas, I., Sahin, O. (2018). Dynamic Pricing and Replenishment with Customer Upgrades. Production and Operations Management, 27(4), 663-679.

Chan R., Li Z. \& Matsypura D. (2020). Assortment optimisation problem: A distribution-free approach. Omega, 95, Accepted for publication.

Chand, S., Ward, J.E., \& Weng, Z.K. (1994). A parts selection model with one-way substitution. European Journal of Operational Research, 73(1), 65-69.

Chanda, U., Bardhan, A.K. (2008). Modelling innovation and imitation sales of products with multiple technological generations. Journal of High Technology Management Research, 18(2), 173-190

Chanda, U. (2011). The dynamic price-quality decision model for two successive generations of technology innovation. International Journal of Innovation and Technology Management, 8(4), 635-660.

Chanda, U. \& Aggarwal, R. (2014). Optimal inventory policies for successive generations of a high technology product. Journal of High Technology Management Research, 25(2), 148-162.

Chanda, U. \& Das, S. (2015). Multi-stage diffusion dynamics in multiple generation high technology products. Journal of High Technology Management Research, 26(1), 88-104.

Chen, B. and Chao, X. and Ahn, H.S. (2015). Coordinating Pricing and Inventory Replenishment with Nonparametric Demand Learning. Ross School of Business Paper No. 1294.

Chen X., Feng Y., Keblis M.F., Xu J. (2015). Optimal inventory policy for two substitutable products with customer service objectives. European Journal of Operational Research, 246(1), 76-85.

Chen, B. \& Chao, X. (2020). Dynamic Inventory Control with Stockout Substitution and Demand Learning. Management Science, Accepted for Publication

Chen, L. \& Plambeck, E.L. (2008). Dynamic Inventory Management with Learning About Demand Distribution and Substitution Probability. Manufacturing and Service Operations Management, 10(2), 236-256.

Chiang, W. K. (2010). Product availability in competitive and cooperative dual-channel distribution with stock-out based substitution. European Journal of Operational Research, 200(1), 111-126.

Chopra, S., \& Meindl, P. (2010). Supply chain management: Strategy, planning, and operation (4th ed.). New Jersey: Prentice Hall.

Cosgun O., Kula U., Kahraman, C. (2017). Markdown optimization for an apparel retailer under cross-price and initial inventory effects. Knowledge-Based Systems, 120, 186-197.

Danaher, P.J., Hardie, B.G.S., \& Putsis Jr., W.P. (2001). Marketingmix variables and the diffusion of successive generations of a technological innovation. Journal of Marketing Research, 38(4), 501-514. doi:10.1509/jmkr.38.4.501.18907

Dawande, M., Gavirneni, S. Mu, Y., Sethi, S. \& Sriskandarajah, C. (2010). On the Interaction Between Demand Substitution and Production Changeovers. Manufacturing and Service Operations Management, 12(4), 682-691.

Deflem, Y., \& Van Nieuwenhuyse, I. (2013). Managing inventories with one-way substitution: A newsvendor analysis. European Journal of Operational Research, 228(3), 484-493.

Deniz, B., Karaesmen, I., \& Scheller-Wolf, A. (2009). Managing perishables with substitution: Inventory issuance and replenishment heuristics. Manufacturing \& Service Operations Management, 12(2), 319-329.
Desir, A., Goyal, V., Segev, D. \& Ye, C. (2020). Constrained assortment optimization under the Markov chain-based choice model. Management Science, 66(2), 698-721.

Dong, L., Kouvelis, P., \& Tian, Z. (2009). Dynamic pricing and inventory control of substitute products. Manufacturing \& Service Operations Management, 11(2), 317-339.

Dong, Z.S., Chen, W., Zhao, Q., Li, J. (2020). Optimal pricing and inventory strategies for introducing a new product based on demand substitution effects. Journal of Industrial and Management Optimization, 16(2), 725-739

Drezner, Z., Gurnani, H., \& Pasternack, B. A. (1995). An EOQ model with substitutions between products. The Journal of the Operational Research Society, 46(7), 887-891.

Duenyas, I., \& Tsai, C. Y. (2000). Control of a manufacturing system with random product yield and downward substitutability. IIE Transactions, 32(9), 785-795.

Dutta, P. \& Chakraborty, D. (2010). Incorporating one way substitution policy into the newsboy problem with imprecise customer demand. European Journal of Operational Research, 200(1), 99-110.

Ernst, R., \& Kouvelis, R. (1999). The effects of selling packaged goods on inventory decisions. Management Science, 45(8), $1142-1155$.

Etebari, F. (2020). Pricing competition under specific discrete choice models. Asia-Pacific Journal of Operational Research, 37(2), 2050008

Fadiloglu, M., Karasan, O., \& Pinar, M. (2010). A model and case study for efficient shelf usage and assortment analysis. Annals of Operations Research, 180(1), 105-124.

Farahat A., Lee J. (2018). The multiproduct newsvendor problem with customer choice. Operations Research, 66(1), 123-136.

Feng Y., Xu J., Zheng S. (2019). Technical note-dynamic optimal policy for an inventory system of two substitutable products with positive replenishment lead times. Operations Research, 67(4), 1027-1034.

Fisher, M., Vaidyanathan, R. (2014). A demand estimation procedure for retail assortment optimization with results from implementations. Management Science, 60(10), 2401-2415.

Fu K., Gong X., Liang G. (2019). Managing Perishable Inventory Systems with Product Returns and Remanufacturing. Production and Operations Management, 28(6), 1366-1386.

Ganesh, M., Raghunathan, S., \& Rajendran, C. (2008). The value of information sharing in a multi-product supply chain with product substitution. IIE Transactions, 40(12), 1124-1140.

Ganesh, M., Raghunathan, S. \& Rajendran, C. (2014). The value of information sharing in a multi-product, multi-level supply chain: Impact of product substitution, demand correlation, and partial information sharing. Decision Support Systems, 58, 79 94

Gaur, V., \& Honhon, D. (2006). Assortment planning and inventory decisions under a locational choice model. Management Science, 52(10), 1528-1543.

Geunes, J. \& Su, Y. (2019). Single-period assortment and stocklevel decisions for dual sales channels with capacity limits and uncertain demand. International Journal of Production Research, Under Press.

Gilland, W. G., \& Heese, H. S. (2013). Sequence matters: Shelfspace allocation under dynamic customer-driven substitution. Production and Operations Management, 22(4), 875-887.

Goyal, M. \& Netessine, S. (2011). Volume Flexibility, Product Flexibility, or Both: The Role of Demand Correlation and Product Substitution. Manufacturing and Service Operations Management, 13(2), 180-193.

Goyal, V., Levi, R. \& Segev, D. (2016). Near-Optimal Algorithms for the Assortment Planning Problem Under Dynamic Substitution and Stochastic Demand. Operations Research, 64(1), https://doi.org/10.1287/opre.2015.1450 
Gurler, U. \& Yilmaz, A. (2010). Inventory and coordination issues with two substitutable products. Applied Mathematical Modelling, 34(3), 539-551.

Gurler, U., Oztop, S., \& Sen, A. (2009). Optimal bundle formation and pricing of two products with limited stock. International Journal of Production Economics, 118(2), 442462.

Gurnani, H., \& Drezner, Z. (2000). Deterministic hierarchical substitution inventory models. Journal of the Operational Research Society, 51(1), 129-133.

Honhon, D., Gaur, V., \& Seshadri, S. (2010). Assortment planning and inventory decisions under stockout-based substitution. Operations Research, 58(5), 1364-1379.

Honhon, D., \& Seshadri, S. (2013). Fixed vs. random proportions demand models for the assortment planning problem under stockout-based substitution. Manufacturing \& Service Operations Management, 15(3), 378-386.

Hopp, W. J., \& Xu, X. (2005). Product line selection and pricing with modularity in design. Manufacturing \& Service Operations Management, 7(3), 172-187.

Hopp, W. J., \& Xu, X. (2008). A static approximation for dynamic demand substitution with applications in a competitive market. Operations Research, 56(3), 630-645.

Hsieh, C.C., \& Wu, C.H. (2009). Coordinated decisions for substitutable products in a common retailer supply chain. European Journal of Operational Research, 196(1), 273288.

Hsieh, C.C. \& Lai, H.H. (2020). Pricing and ordering decisions in a supply chain with downward substitution and imperfect process yield. Omega, 95,102064.

Hsu, A., \& Bassok, Y. (1999). Random yield and random demand in a production system with downward substitution. Operations Research, 47(2), 277-290.

Hsu, V., Li, C.L., \& Xiao, W.Q. (2005). Dynamic lot size problems with one-way product substitution. IIE Transaction, 37(3), 201-215.

Huang, D., Zhou, H., \& Zhao, Q.H. (2011). A competitive multiple-product newsboy problem with partial product substitution. Omega, 39(3), 302-312.

Huddiniah, E.R. \& E.R., M. (2018). Product Variety, Supply Chain Complexity and the Needs for Information Technology: A Framework Based on Literature Review. Operations and Supply Chain Management, 12(4).

Inderfurth, K. (2004). Optimal policies in hybrid manufacturing/ remanufacturing systems with product substitution. International Journal of Production Economics, 90, 325-343

Ishichi, K., Ohmori, S., Ueda, M. \& Yoshimoto, K. (2019). Shelfspace Allocation Model with Demand Learning. Operations and Supply Chain Management, 12(1), 24-30.

Islam, T., \& Meade, N. (1997). The diffusion of successive generations of a technology: A more general model. Technological Forecasting and Social Change, 56(1), 49-60. doi:10.1016/s0040-1625(97)00030-9.

Jiang, Z. \& Jain, D.C. (2012). A generalized Norton-Bass model for multi-generation diffusion. Management Science, 58(10), 1887-1897

Jing F., Mu Y. (2020). Dynamic lot-sizing model under perishability, substitution, and limited storage capacity. Computers and Operations Research, 122, Accepted for publication.

Jing F., Mu Y. (2019). Forecast horizon for dynamic lot sizing model under product substitution and perishable inventories. Computers and Operations Research, 110, 77-87.

Karakul, M. (2008). Joint pricing and procurement of fashion products in the existence of clearance markets. International Journal of Production Economics, 114(2), 487-506.

Karakul, M., \& Chan, L.M.A. (2008). Analytical and managerial implications of integrating product substitutability in the joint pricing and procurement problem. European Journal of Operational Research, 190(1), 179-204.

Karakul, M., \& Chan, L.M.A. (2010). Joint pricing and procurement of substitutable products with random demands - A technical note. European Journal of Operational Research, 201(1), 324-328.

Khademi A., Eksioglu B. (2018). Spare parts inventory management with substitution-dependent reliability. INFORMS Journal on Computing, 30(3), 507-521.

Khouja, M., Mehrez, A., \& Rabinowitz, G. (1996). A two-item newsboy problem with substitutability. International Journal of Production Economics, 44(3), 267-275.

Kim, N., Chang, D.R., \& Shocker, A.D. (2000). Modeling intercategory and generational dynamics for a growing information technology industry. Management Science, 46(4), 496-512. doi:10.1287/mnsc.46.4.496.12059.

Kim S.W., Bell P.C. (2019). Stochastic optimization models with substitution as a result of price differences and stockouts. International Transactions in Operational Research, 26(6), 2129-2151.

Kim S.W., Bell P.C. (2015). A note on the optimal pricing and production decisions with price-driven substitution. International Transactions in Operational Research, 22(6), 1097-1116.

Kim, S.W. \& Bell, P.C. (2011). Optimal pricing and production decisions in the presence of symmetrical and asymmetrical substitution. Omega, 39(5), 528-538.

Kok, A. G., \& Fisher, M. L. (2007). Demand estimation and assortment optimization under substitution: Methodology and application. Operations Research, 55(6), 1001-1021.

Kraiselburd, S., Narayanan, V. G., \& Raman, A. (2004). Contracting in a supply chain with stochastic demand and substitute products. Production and Operations Management, 13(1), 46-62.

Krommyda, I.P., Skouri, K. \& Konstantaras, I. (2015). Optimal ordering quantities for substitutable products with stockdependent demand. Applied Mathematical Modelling, 39(1), 147-164.

Lang, J. C., \& Domschke, W. (2010). Efficient reformulations for dynamic lot-sizing problems with product substitution. $O R$ Spectrum, 32(2), 263-329.

Li, Q. \& Ha, A.Y. (2008). Reactive capacity and inventory competition under demand substitution, IIE Transactions, 40(8), 707-717.

Li, Y., Chen, J., \& Cai, X. (2006). Uncapacitated production planning with multiple product types, returned product remanufacturing, and demand substitution. OR Spectrum, 28(1), 101-125.

Li, Z. (2007). A single-period assortment optimization model. Production and Operations Management, 16(3), 369-380.

Li, Z. \& Fu, Q.G. (2017). Robust inventory management with stock-out substitution. International Journal of Production Economics, 193, 813-826.

Lin, K.Y., Sibdari, S.Y. (2009). Dynamic price competition with discrete customer choices. European Journal of Operational Research, 197(3), 969-980.

Lippman, S. A., \& McCardle, K. F. (1997). The competitive newsboy. Operations Research, 45(1), 54-65.

Liu, J., \& Lee, C. (2007). Evaluation of inventory policies with unidirectional substitutions. European Journal of Operational Research, 182(1), 145-163.

Ma, S., Sahin, E., Dallery, Y. \& Jemai, Z. (2015). Assortment decision in the multi-product News-Vendor Problem with demand substitution, 2015 International Conference on Industrial Engineering and Systems Management), Seville, 304-311, DOI: 10.1109/IESM.2015.7380176.

Maddah, B., \& Bish, E.K. (2007). Joint pricing, assortment, and inventory decisions for a retailer's product line. Naval Research Logistics, 54(3), 315-330. 
Maddah, B., Bish, E.K. \& Tarhini, H. (2014). Newsvendor pricing and assortment under Poisson decomposition. IIE Transactions, 46(6), 564-584.

Mahajan, S., \& van Ryzin, G. (2001a). Stocking retail assortments under dynamic consumer substitution. Operations Research, 49(3), 334-351.

Mahajan, S., \& van Ryzin, G. (2001b). Inventory competition under dynamic consumer choice. Operations Research, 49(5), 646-657.

Majumder P., Bera U.K., Maiti M. (2020). An EPQ model of substitutable products under trade credit policy with stock dependent and random substitution. OPSEARCH, Accepted for publication

McGillivary, A.R., \& Silver, E.A. (1978). Some concepts for inventory control under substitutable demand. INFOR, 16(1), 47-63.

McGuire, T.W., \& Staelin, R. (1983). An industry equilibrium analysis of downstream vertical integration. Marketing Science, 2(2), 161-191.

Mosenson, R., \& Dror, E. (1972). A Solution to the Qualitative Substitution Problem in Demand Theory. The Review of Economic Studies, 39(4), 433-441

Mukhopadhyay A., Goswami A. (2017). An inventory model with shortages for imperfect items using substitution of two products. International Journal of Operational Research, 30(2), 193-219.

Nagarajan, M., \& Rajagopalan, S. (2008). Inventory models for substitutable products: Optimal policies and heuristics. Management Science, 54(8), 1453-1466.

Nagasawa, K., Irohara, T., Matoba, Y., \& Liu, S. (2014). Joint Replenishment Problem in Multi-Item Inventory Control with Carrier Capacity and Receiving Inspection Cost. Operations and Supply Chain Management: An International Journal, 6(3), 111-116.

Netessine, S., Dobson, G., \& Shumsky, R. A. (2002). Flexible service capacity: Optimal investment and the impact of demand correlation. Operations Research, 50(2), 375-388.

Netessine, S., \& Rudi, N. (2003). Centralized and competitive inventory models with demand substitution. Operations Research, 51(2), 329-335.

Newman, J.P., Ferguson, M.E., Garrow, L.A. \& Jacobs, T.L. (2014). Estimation of choice-based models using sales data from a single firm. Manufacturing and Service Operations Management, 16(2), 184-197.

Norton, J.A., \& Bass, F.M. (1987). A diffusion theory model of adoption and substitution for successive generations of hightechnology products. Management Science, 33(9), 1069-1086.

Pan, Q.H., He, X., Skouri, K., Chen, S.C., Teng, J.T. (2018). An inventory replenishment system with two inventory-based substitutable products. International Journal of Production Economics, 204, 135-145.

Pan, X.A., \& Honhon, D. (2012). Assortment planning for vertically differentiated products. Production and Operations Management, 21(2), 253-275.

Parlar, M. (1988). Game theoretic analysis of the substitutable product inventory problem and random demands. Naval Research Logistics, 35(3), 397-409.

Pasternack, B.A., \& Drezner, Z. (1991). Optimal inventory policies for substitutable commodities with stochastic demand. Naval Research Logistics, 38(2), 221-240.

Pentico, D.W. (1974). The assortment problem with probabilistic demands. Management Science, 21(3), 286-290.

Pentico, D.W. (1976). The assortment problem with nonlinear cost functions. Operations Research, 24(6), 1129-1142.

Pentico, D.W. (1988). The discrete two dimensional assortment problem. Operations Research, 36(2), 324-332.

Pineyro, P., \& Viera, O. (2010). The economic lot-sizing problem with remanufacturing and one-way substitution. International Journal of Production Economics, 124(2), 482-488.
Rajaram, K. \& Tang, C.S. (2001). The impact of product substitution on retail merchandising. European Journal of Operational Research, 135(3), 582-601.

Rao, U.S., Swaminathan, J.M. \& Zhang, J. (2004). Multi-product inventory planning with downward substitution, stochastic demand and setup costs. IIE Transactions, 36(1), 59-71, 10.1080/07408170490247304

Rasouli N., Amin-Naseri M.R., Zaccour G. (2020). Pricing and order quantity of substitutes in two inventory-related markets. International Transactions in Operational Research, Accepted for publication.

Rusmevichientong, P. \& Shen, Z.J.M. \& Shmoys, D.B. (2010). Dynamic assortment optimization with a multinomial logit choice model and capacity constraint. Operations Research, 58(6), 1666-1680.

Sainathan, A. (2013). Pricing and Replenishment of Competing Perishable Product Variants under Dynamic Demand Substitution. Production and Operations Management, 22(5), 1157-1181.

Saure, D. \& Zeevi, A. (2013). Optimal dynamic assortment planning with demand learning. Manufacturing and Service Operations Management, 15(3), 387-404.

Schlapp J., Fleischmann M. (2018). Technical note - Multiproduct inventory management under customer substitution and capacity restrictions. Operations Research, 66(3), 740-747.

Serin, Y. (2007). Competitive newsvendor problems with the same Nash and Stackelberg solutions. Operations Research Letters, 35(1), 83-94.

Shah, J. \& Avittathur, B. (2007). The retailer multi-item inventory problem with demand cannibalization and substitution. International Journal of Production Economics, 106(1), 104114.

Shah, N.H., Chaudhari, U. \& Jani, M.Y. (2019). Inventory Control Policies for Substitutable Deteriorating Items Under Quadratic Demand. Operations and Supply Chain Management, 12(1), 4248.

Shumsky, R.A. \& Zhang, F. (2009). Dynamic Capacity Management with Substitution. Operations Research, 57(3), https://doi.org/10.1287/opre.1080.0610

Sivakumar, B. (2008). Two-commodity inventory system with retrial demand. European Journal of Operational Research, 187(1), 70-83

Smith, S.A., \& Agrawal, N. (2000). Management of Multi-Item Retail Inventory Systems with Demand Substitution. Operations Research, 48(1), 50-64.

Snyder, H. (2019). Literature Review as a research methodology: An overview and guidelines. Journal of Business Research, 104, 333-339.

Song, J. S., \& Xue, Z. (2007). Demand management and inventory control for substitutable products. Working paper.

Stavrulaki, E. (2011). Inventory decisions for substitutable products with stock-dependent demand. International Journal of Production Economics, 129(1), 65-78.

Suh, M. \& Aydin, G. (2011). Dynamic pricing of substitutable products with limited inventories under logit demand. IIE Transactions, 43(5), 323-331

Surti C., Abad P., Hassini E. (2018). Impact of inventory cannibalisation on a retailer selling substitutes. International Journal of Operational Research, 33(2), 179-207.

Tan, B., \& Karabati, S. (2013). Retail inventory management with stock-out based dynamic demand substitution. International Journal of Production Economics, 145(1), 78-87.

Tang, C.S. \& Yin, R. (2009). Joint Ordering and Pricing Strategies for Managing Substitutable Products. Production and Operations Management, 16(1), 138-153.

Tang, C.S., \& Yin, R. (2010). The implications of costs, capacity, and competition on product line selection. European Journal of Operational Research, 200(2), 439-450. 
Timmermans, H., \& Golledge, R. G. (1990). Applications of behavioural research on spatial problems II: preference and choice. Progress in human geography, 14(3), 311-354.

Torraco, R.J. (2005). Writing integrated literature reviews: guidelines and examples. Human Resource Development Review, 4(3), 356-367.

Transchel S. (2017). Inventory management under price-based and stockout-based substitution. European Journal of Operational Research, 262(3), 996-1008.

Vaagen, H., Wallace, S.W., \& Kaut, M. (2009). Modelling consumer-directed substitution. International Journal of Production Economics, 134(2), 388-397.

Van Ryzin, G., \& Mahajan, S. (1999). On the relationship between inventory costs and variety benefits in retail assortments. Management Science, 45(11), 1496-1509.

Wan M., Huang Y., Zhao L., Deng T., Fransoo J.C. (2018). Demand estimation under multi-store multi-product substitution in high density traditional retail. European Journal of Operational Research, 266(1), 99-111.

Wei, J., Zhao, J. (2016). Pricing decisions for substitutable products with horizontal and vertical competition in fuzzy environments. Annals of Operations Research, 242(2), 505528

Wu, C., Petruzzi, N.C., \& Chhajed, P. (2007). Vertical integration with price-setting competitive newsvendors. Decision Science, 38(4), 581-610.

Xia, Y. (2011). Competitive strategies and market segmentation for suppliers with substitutable products. European Journal of Operational Research, 210(2), 194-203.

$\mathrm{Xu}, \mathrm{H}$., Yao, D.D., \& Zheng, S. (2010). Optimal control of replenishment and substitution in an inventory system with nonstationary batch demand. Production and Operations Management, 20(5), 727-736.

Xu, H., Yao, D.D. \& Zheng, S. (2016). Optimal policies for a twoproduct inventory system under a flexible substitution scheme. Production and Operations Management, 25(6), 1088-1105.

Xu, X., Li, Y. \& Cai, X. (2012). Optimal policies in hybrid manufacturing/remanufacturing systems with random price- sensitive product returns. International Journal of Production Research, 50(23), 6978-6998.

Yadavalli, V.S.S., Van Schoor, C.D.W., \& Udayabaskaran, S (2006). A substitutable two-product inventory system with joint-ordering policy and common demand. Applied Mathematics and Computation, 172(2), 1257-1271.

Yang, H.S., \& Schrage, L. (2009). Conditions that cause risk pooling to increase inventory. European Journal of Operational Research, 192(3), 837-851.

Yu, Y., Shou, B., Ni, Y., Chen, L. (2017). Optimal production, pricing, and substitution policies in continuous review production-inventory systems. European Journal of Operational Research, 260(2), 631-649.

Yucel, E., Karaesmen, F. Salman, F.S. \& Turkay, M. (2009). Optimizing product assortment under customer-driven demand substitution. European Journal of Operational Research, 199(3), 759-768.

Yudhistyra, W.I., Risal, E.M., Raungratanaamporn, I. \& Ratanavaraha, V. (2020). Exploring Big Data Research: A Review of Published Articles from 2010 to 2018 Related to Logistics and Supply Chains. Operations and Supply Chain Management, 13(2), 134-149.

Zeppetella, L., Gebennini, E., Grassi A. \& Rimini, B. (2017). Optimal production scheduling with customer-driven demand substitution. International Journal of Production Research, 55(6), 1692-1706.

Zhang, Y., Hua, G., Wang, S., Zhang, J. \& Fernandez V. (2018). Managing demand uncertainty: Probabilistic selling versus inventory substitution. International Journal of Production Economics, 196, 56-67.

Zhao, J., Tang, W., Zhao, R. \& Wei, J. (2012a). Pricing decisions for substitutable products with a common retailer in fuzzy environments. European Journal of Operational Research, $216(2), 409-419$.

Zhao, J., Tang, W. \& Wei, J. (2012b). Pricing decision for substitutable products with retail competition in a fuzzy environment. International Journal of Production Economics, $135(1), 144-153$.

Gaurav Nagpal is an Assistant Professor in Management Department of Birla Institute of Technology and Science Pilani. His area of expertise is Operations and Supply Chain Management, Operations Research, Industrial Engineering, Business Statistics and Data Analytics. He completed his MBA from Indian Institute of Foreign Trade, New Delhi. He has been a GATE topper with 99.83 percentile (AIR-41) in mechanical engineering. He has also done EPMBD (one year Executive Program in Managing Business Decisions) from IIM Calcutta, and is CSCP (Certified Supply Chain Professional) qualified from APICS. He has twelve years of industry experience and has worked for the leading grocery e-Commerce Indian firm "Grofers" as Associate Director- Business Planning and Analysis.

Udayan Chanda is currently working as Associate Professor in Department of Management, Birla Institute of Technology \& Science (BITS) Pilani. Earlier he was associated with Industrial Statistics Lab., Department of Information \& Industrial Engineering Yonsei University as Post Doctoral Fellow and Department of Operational Research, University of Delhi as Assistant Professor (Ad-hoc). He received his Ph.D. degree in Marketing Models and Optimization (Operational Research) from University of Delhi, Delhi. He has published numerous papers in the area of Marketing Models, Optimization, Software Reliability and Inventory Management in international journals and conference proceedings. His current research interests include Marketing Models, Inventory Modelling, Software Reliability Growth Modelling, and Dynamic Optimization Techniques. He has around 50 research publications in reputed journals to his credit.

Himanshu Seth is a research scholar in department of management, BITS Pilani. His research area includes working capital management. Additionally, he also specializes in data development analysis, efficiency and performance management. He has published several papers in journal of national and international repute. 